\title{
The post-common envelope central stars of the planetary nebulae Henize 2-155 and Henize 2-161*
}

\author{
D. Jones ${ }^{1,2}$, H. M. J. Boffin ${ }^{3}$, P. Rodríguez-Gil ${ }^{1,2}$, R. Wesson ${ }^{3}$, R. L. M. Corradi ${ }^{1,2}$, B. Miszalski ${ }^{4,5}$, and S. Mohamed ${ }^{4}$
}

1 Instituto de Astrofísica de Canarias, 38200 La Laguna, Tenerife, Spain e-mail: djones@iac.es

2 Departamento de Astrofísica, Universidad de La Laguna, 38206 La Laguna, Tenerife, Spain

3 European Southern Observatory, Alonso de Córdova 3107, Casilla 19001, Santiago, Chile

${ }^{4}$ South African Astronomical Observatory, PO Box 9, Observatory, 7935 Cape Town, South Africa

5 Southern African Large Telescope Foundation, PO Box 9, Observatory, 7935 Cape Town, South Africa

Received 3 December 2014 / Accepted 5 June 2015

\section{ABSTRACT}

\begin{abstract}
We present a study of Hen 2-155 and Hen 2-161, two planetary nebulae which bear striking morphological similarities to other planetary nebulae known to host close-binary central stars. Both central stars are revealed to be photometric variables while spectroscopic observations confirm that Hen 2-155 is host to a double-eclipsing, post-common-envelope system with an orbital period of $3^{\mathrm{h}} 33^{\mathrm{m}}$ making it one of the shortest period binary central stars known. The observations of Hen 2-161 are found to be consistent with a post-common-envelope binary of period $\sim 1$ day. A detailed model of the central star of Hen 2-155 is produced, showing the nebular progenitor to be a hot, post-AGB remnant of approximately $0.62 M_{\odot}$, consistent with the age of the nebula, and the secondary star to be an M dwarf whose radius is almost twice the expected zero age main sequence radius for its mass. In spite of the small numbers, all main-sequence companions, of planetary nebulae central stars, to have had their masses and radii constrained by both photometric and spectroscopic observations have also been found to display this "inflation". The cause of the "inflation" is uncertain but is probably related to rapid accretion, immediately before the recent common-envelope phase, to which the star has not yet thermally adjusted. The chemical composition of both nebulae is also analysed, showing both to display elevated abundance discrepancy factors. This strengthens the link between elevated abundance discrepancy factors and close binarity in the nebular progenitor.
\end{abstract}

Key words. binaries: eclipsing - binaries: close - circumstellar matter - planetary nebulae: individual: Henize 2-155 planetary nebulae: individual: Henize 2-161

\section{Introduction}

Planetary nebulae (PNe) with close binary central stars offer an important laboratory for the study of binary evolution - in particular, the poorly understood common envelope (CE) phase. To date, approximately 50 close binary central stars (hereafter bCSPNe - binary central stars of PNe) have been discovered $^{1}$ (Miszalski et al. 2009a; De Marco et al. 2008; Boffin $2014 b$ ) with many more expected (given the high binary fraction amongst those nebulae observed by photometric surveys, e.g. Miszalski et al. 2009a; Bond 2000). Finding, and accurately constraining the orbital parameters of, these missing binaries is a time consuming task, with a completely unbiased survey requiring many, many nights of photometric monitoring and subsequent spectroscopic follow-up. As such, it is more reasonable to use the morphological features identified by Miszalski et al. (2009b), as being particularly prevalent amongst PNe with binary central stars, as selection criteria for targeted photometric searches for new binaries. These features include filamentary structures, rings, polar outflows and bipolarity, and have been successfully employed by our group in the discovery of several new binary central stars (Miszalski et al. 2011a,b; Corradi et al. 2011; Boffin et al. 2012b; Jones et al. 2014a; Santander-García et al. 2015).

\footnotetext{
* Full Tables 2-5, and 7 are only available at the CDS via anonymous ftp to cdsarc.u-strasbg. fr (130.79.128.5) or via http://cdsarc.u-strasbg.fr/viz-bin/qcat?J/A+A/580/A19 1 http://drdjones.net/bCSPN
}

Here, we report on the discovery of post-common envelope central stars at the heart of the PNe Hen 2-155 and Hen 2-161, two nebulae selected for study based on their similar appearance to other PNe known to host binary central stars. We also highlight the novel use of narrowband filters in our photometric monitoring, which afford the possibility to study some of the bright nebulae previously inaccessible to broadband photometric methods.

Hen 2-155 $\left(\alpha=16^{\mathrm{h}} 19^{\mathrm{m}} 23.17^{\mathrm{s}}, \delta=-42^{\circ} 15^{\prime} 36.64^{\prime \prime}\right.$, PN G338.8+05.6) and Hen 2-161 $\left(\alpha=16^{\mathrm{h}} 24^{\mathrm{m}} 37.79^{\mathrm{s}}, \delta=\right.$ $-53^{\circ} 22^{\prime} 34.14^{\prime \prime}$, PN G331.5-02.7) were both discovered by Karl G. Henize in 1967, forming part of his catalogue of southern PNe (Henize 1967). Both PNe display elongated (following the classification scheme of Sahai et al. 2011) morphologies with knotty filamentary waists, typical of those PNe already known to host binary central stars. Beyond this initial similarity, both nebulae are remarkably similar to some of the most interesting examples in the currently known sample and were, therefore, selected by our group for study.

The wispy extensions and irregular central filaments of Hen 2-155 (see Fig. 1) are extremely similar to those found in NGC 6326, NGC 6778, Hen 2-11, and NGC 5189 (Miszalski et al. 2011b; Jones et al. 2014a; Manick et al. 2015). The geometrically opposed pairs of "knots" seen marking the ends of the major axis of the nebula are relatively typical of high velocity outflows or "jets" which are believed to be typical of central star binarity. Of additional interest, the central binaries of NGC 6326, NGC 6778 and Hen 2-11 all display extremely 
A\&A 580, A19 (2015)

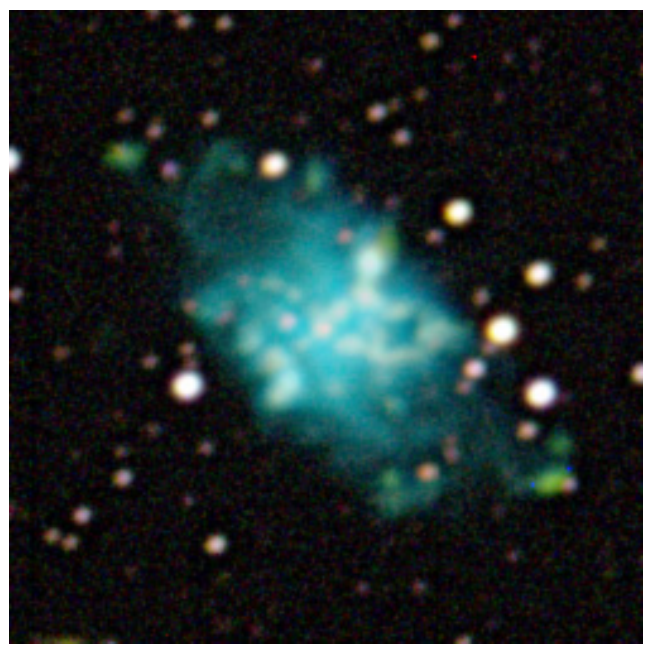

Fig. 1. Colour composite image of Hen 2-155 (for exposures in individual filters please see Fig. 3). North is up, east is to the left. The image measures $1^{\prime} \times 1^{\prime}$.

strong photometric variability and periods shorter than 1 day (much shorter in the case of NGC 6778 at 0.1534 day), making for relatively easy detection, whilst Hen 2-11 and NGC 6778 are known to host eclipsing systems. Eclipses are key in measuring accurate and model independent masses (Miszalski et al. 2008), which are, in turn, extremely important in accurately constraining the state of these binaries upon exiting the CE. The presence of the PN ensures that the system is "fresh out of the oven" as the nebula itself is the product of the ejection of the CE, and the lifetime of the PN is insufficient for significant changes to have occurred in the binary post-ejection.

Hen 2-161 shows a knotty equatorial ring forming the waist of an elongated, possibly bipolar, morphology (Fig. 2 of this paper, and figure 3 lower panel of Sahai et al. 2011) reminiscent of the morphology of the Necklace (Fig. 2), as well as several other post-CE PNe (e.g. Sab 41, Miszalski et al. 2009b; and Fg 1, Boffin et al. 2012b). Once again, the central binary of the Necklace shows extremely strong variability and a relatively short period (1.16 day, Corradi et al. 2011), but is of special interest due to the chemical peculiarity of the companion star in the system. The secondary in the Necklace has been shown to be a carbon dwarf star, indicating that it has been chemically polluted (with asymptotic giant branch (AGB) material) by the primary star - thus far the only direct evidence of mass transfer during or, much more likely, just prior to the CE phase in a PN (Miszalski et al. 2013). Additionally, the central star of Hen 2-161 has been shown to be displaced from the geometric centre of the nebula (Sahai et al. 2011), another possible indicator of central star binarity (Soker et al. 1998).

A summary of the known parameters, stellar and nebular, of both Hen 2-155 and Hen 2-161 can be found in Table 1.

The paper is structured as follows. Sect. 2 outlines the observations and data reduction, Sect. 3 describes the light curve analysis and modelling, and the analysis of the stellar and nebular spectroscopy. Finally, Sect. 4 discusses the results.

\section{Observations and data reduction}

\subsection{Photometry}

Photometric monitoring of the central stars of Hen 2-155 and Hen 2-161 was carried out using the EFOSC2 instrument on the 3.6-m ESO-NTT (Buzzoni et al. 1984; Snodgrass et al. 2008). The observations employed the E2V CCD with a pixel scale
Table 1. Parameters, both stellar and nebular, of Hen 2-155 and Hen 2-161 from the literature.

\begin{tabular}{rccc}
\hline \hline & Hen 2-155 & Hen 2-161 & References \\
\hline Reddening, $c\left(\mathrm{H}_{\beta}\right)$ & $0.81-1.01$ & $0.35-1.80$ & 1,2 \\
Distance $(\mathrm{kpc})$ & $2.47-3.15$ & $3.64-5.31$ & 3,4 \\
Central star temperature & $T_{Z}(\mathrm{H})=51$ & $T_{E B}=35.1$ & 5,6 \\
Estimate(s) $(\mathrm{kK})$ & $T_{Z}(\mathrm{He} \mathrm{II})=73$ & & \\
Nebular systemic & $-35.3 \pm 15.0$ & $-98.0 \pm 9.0$ & 7 \\
Velocity ( km s $\left.{ }^{-1}\right)$ & & & \\
Approximate & $14.5-16.9^{a}$ & $9.7-16.3$ & 8 \\
Nebular diameter (") & &
\end{tabular}

Notes. ${ }^{(a)}$ This measurement encompasses the "waist" of the nebula but not the wispy extensions which reach out to $\sim 30^{\prime \prime}$ from the central star. References. (1) Cavichia et al. (2010); (2) Cahn et al. (1992); (3) Phillips (2004); (4) Stanghellini et al. (2008); (5) Kaler \& Jacoby (1991); (6) Preite-Martinez et al. (1989); (7) Durand et al. (1998); (8) Tylenda et al. (2003).

of $0.24^{\prime \prime} \times 0.24^{\prime \prime}$ pixel $^{-1}$. Hen $2-155$ was observed with the $\mathrm{H} \beta$-continuum filter (\#743) on the nights of February 27-29, March 1-2 2012, and February 23-26 2014, while Hen 2-161 was observed with the Gunn $i$ filter (\#705) on the nights of February 29, March 1-2 2012, January 14-15, June 2-6 2013, and February 23-26 2014. For both objects, the exposure time was varied in order to ensure a sufficiently high signal-to-noise ratio in the resulting images (for a full list of individual exposures, see Tables 2 and 3 available at the CDS). All data were debiased and flat-fielded using standard STARLINK routines ${ }^{2}$.

Narrowband emission line images of both objects were also acquired with EFOSC2 and the following filters: $\mathrm{H} \alpha$ (\#692, includes [N II] $\lambda 6548 \AA$ ), [O III] $\lambda 5007 \AA$ (\#687) and [S II] $\lambda 6716+6731 \AA$ (\#702), as well as in broadband Bessel $\mathrm{B}$ (\#639), V (\#641), R(\#642), and Gunn $i$ (\#705). A colour composite of Hen 2-155 made from the narrowband exposures is presented in Fig. 1, while examples of the individual exposures in each of the narrowband filters (including $\mathrm{H} \beta$-continuum, where the central star is highlighted) are shown in Fig. 3.

Further images of Hen 2-161 were taken using a Cousins I-band filter and the SAAO CCD instrument mounted on the SAAO 1.0-m telescope on the night of April 2nd 2012. The $1 \mathrm{~K} \times 1 \mathrm{~K}$ STE4 CCD was employed without binning, resulting in a pixel scale of $0.31^{\prime \prime} \times 0.31^{\prime \prime}$ pixel $^{-1}$.

Contamination from high nebular background can make precision photometry of the central star difficult, even leading to spurious variability (often correlated with the seeing; Jones 2011). As such, the choice of filter for monitoring was made based on estimates of the nebular background level with respect to the brightness of the central star in each filter. Previous monitoring campaigns (and surveys) have experienced most success with $I$-band monitoring, as this offers the best combination of broad bandpass (to reduce exposure times) and avoids the brightest nebular emission lines. However, for brighter nebulae with similarly bright central stars (like Hen 2-155), even the $I$-band can show marked nebular emission, in these cases, monitoring can be successfully performed in $\mathrm{H} \beta$-continuum. The $\mathrm{H} \beta$-continuum filter (\#743) excludes all nebular emission lines reducing the nebular contamination purely to continuum emission, offering the opportunity to observe the central star with minimal influence from the nebula. Given the large

http://starlink.jach.hawaii.edu/ 

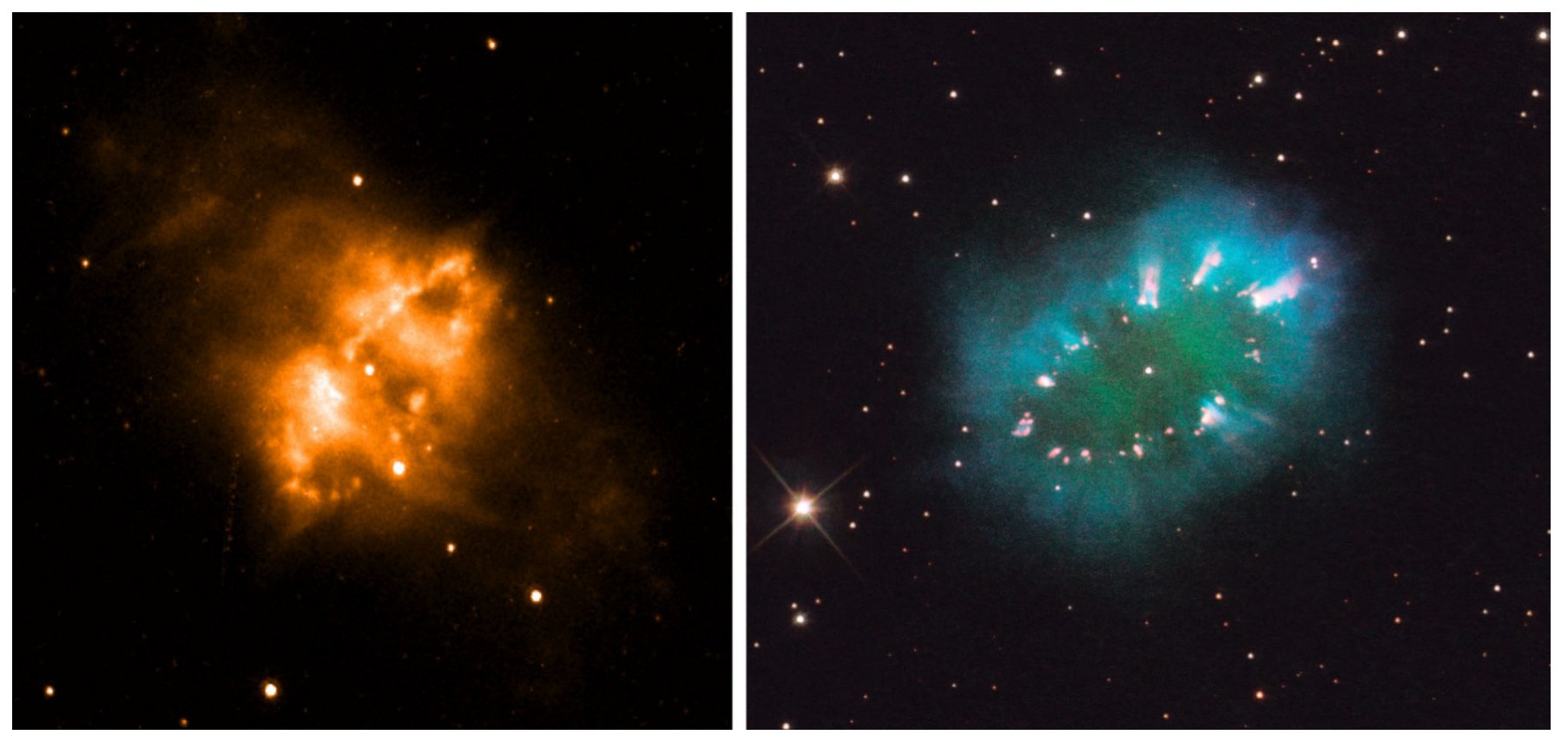

Fig. 2. HST images of Hen 2-161 (left, see also Sahai et al. 2011) and the Necklace (right; Corradi et al. 2011) highlighting their remarkably similar appearances (elongated with knotty waists). The image of Hen $2-161$ is in the light of $\mathrm{H} \alpha+[\mathrm{N}$ II $] \lambda 6584 \AA$, measuring roughly $20^{\prime \prime} \times 20^{\prime \prime}$, while the Necklace image is a colour composite (Credit: NASA, ESA, and the Hubble Heritage Team (STScI/AURA)) measuring roughly 39" $\times 35^{\prime \prime}$. Note that the central star of Hen 2-161 is offset to the northeast from the geometric centre of the nebular ring (in both images, north is up and east is to the left).

Table 2. Excerpt of the log of the $\mathrm{H} \beta$-continuum photometric observations and measurements of the central star of Hen 2-155.

\begin{tabular}{rccc}
\hline \hline Julian date & $\begin{array}{c}\text { Exposure time } \\
(\mathrm{s})\end{array}$ & $\mathrm{H} \beta$-continuum magnitude & $\begin{array}{c}\text { Uncertainty on } \\
\mathrm{H} \beta \text {-continuum magnitude }\end{array}$ \\
\hline 2455985.7989840 & 90 & 15.0020 & 0.0304 \\
2455985.8008033 & 150 & 15.0008 & 0.0211 \\
2455985.8029363 & 150 & 15.0086 & 0.0213 \\
2455985.8050782 & 150 & 15.0051 & 0.0211 \\
2455985.8517651 & 150 & 14.8521 & 0.0193 \\
\hline
\end{tabular}

Notes. Full table available in machine-readable format at the CDS.

Table 3. Excerpt of the log of the $I$-band photometric observations and measurements of the central star of Hen 2-161.

\begin{tabular}{rccc}
\hline \hline Julian date & $\begin{array}{c}\text { Exposure time } \\
(\mathrm{s})\end{array}$ & $I$-band magnitude & $\begin{array}{c}\text { Uncertainty on } \\
I \text {-band magnitude }\end{array}$ \\
\hline 2455987.7904517 & 45 & 15.2006 & 0.0120 \\
2455987.7913786 & 45 & 15.1932 & 0.0121 \\
2455987.7923043 & 45 & 15.2113 & 0.0120 \\
2455987.8456948 & 45 & 15.1422 & 0.0117 \\
2455987.8466095 & 45 & 15.1478 & 0.0116 \\
\hline
\end{tabular}

Notes. Full table available in machine-readable format at the CDS.

aperture of the ESO-NTT and the bright nature of the central star of Hen 2-155, photometric monitoring was possible in $\mathrm{H} \beta$ continuum with high signal-to-noise ratio and with minimal nebular contamination.

Even with the use of filters excluding the majority of the nebular emission, some remains, as such photometry was extracted from both targets with an aperture tailored to a diameter of roughly $3 \times$ the maximum seeing during the observations ( $5^{\prime \prime}$, thus minimising the variable contamination in the aperture; Jones 2011). Photometry was performed using the SEXTRACTOR software (Bertin \& Arnouts 1996), and the differential magnitude of the central stars measured against non-variable field stars. The $I$-band observations of Hen 2-161 were then placed on an absolute scale using catalogue photometry from DENIS (Epchtein et al. 1999) and the methodology described in Boffin et al. (2012a), with an approximate precision of 0.05 mag (derived from the dispersion of detector zero points calculated from each field star). The $\mathrm{H} \beta$-continuum observations of Hen 2-155 were also placed on an approximate absolute scale using standard stars observed during the observing runs (this can only be considered approximate due to the variable nature of extinction during each night and each run, however as the value of each data point is determined by relative brightness to field stars no spurious variability can have been introduced as part of this process). All photometric measurements and their uncertainties are shown in Tables 2 and 3 available at the CDS. 


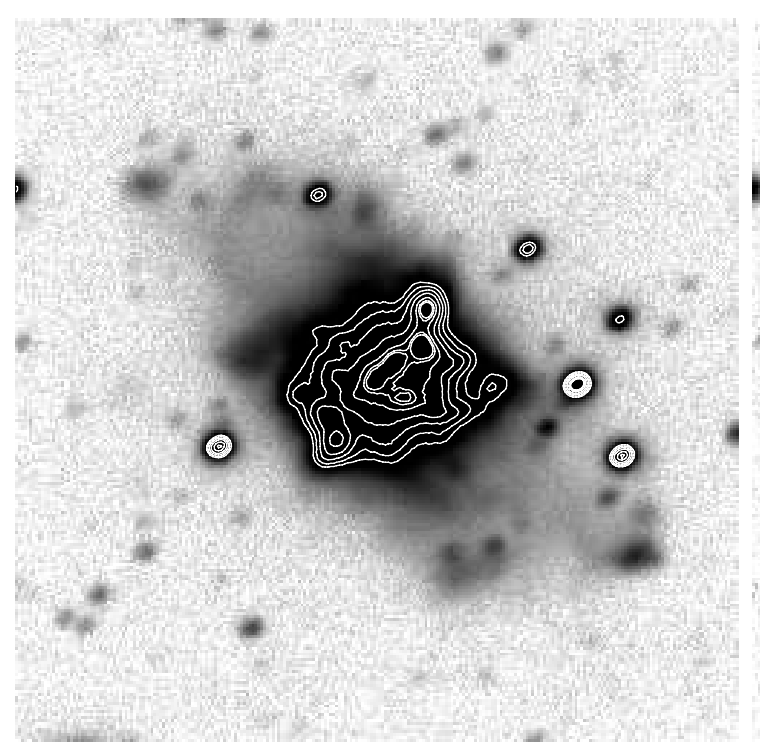

(a)

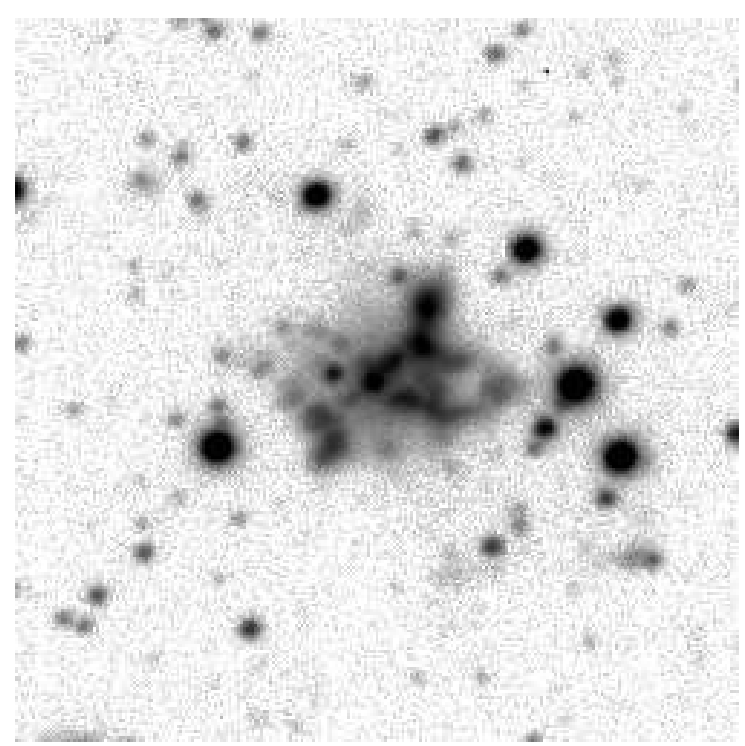

(c)

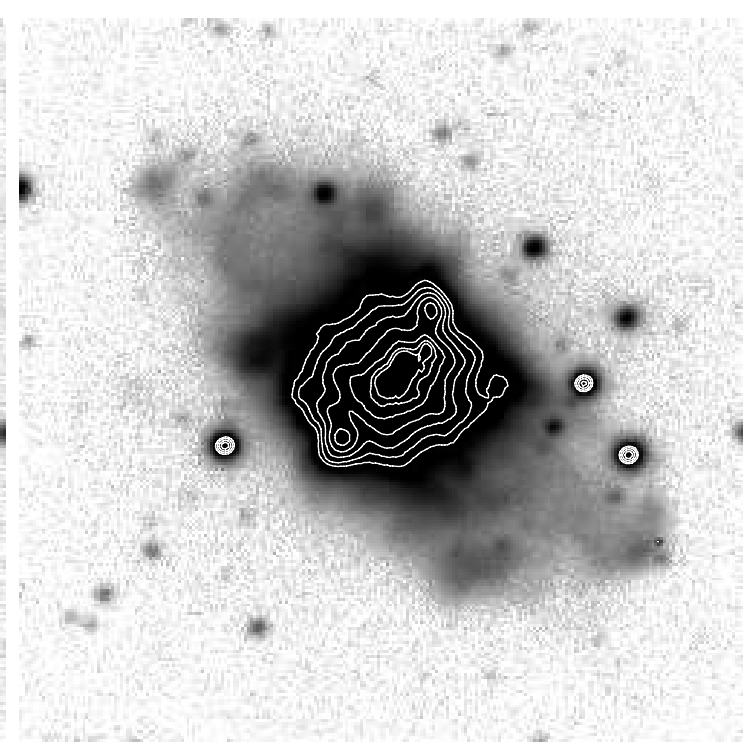

(b)

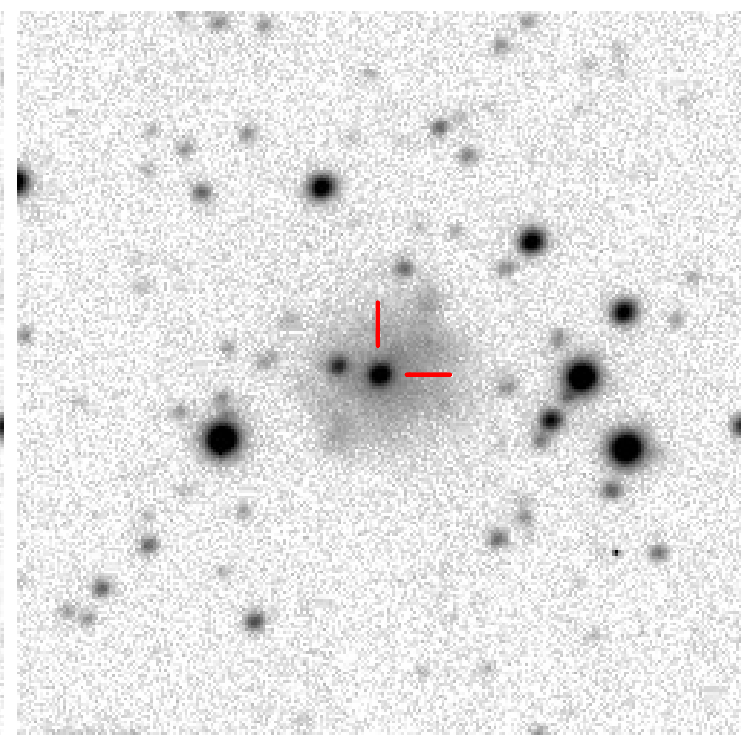

(d)

Fig. 3. NTT-EFOSC 2 images of Hen $2-155$ in the light of a) $\mathrm{H} \alpha+[\mathrm{N} \mathrm{II}]$; b) [O III] $\lambda 5007 \AA$; c) [S II] $\lambda 6716+6731 \AA$ and d) $\mathrm{H} \beta$-continuum (with central star highlighted). North is up, east to the left and each image measures $1^{\prime} \times 1^{\prime}$. All images are displayed on a logarithmic scale. The contours in a) and b) represent the 99.7, 99.5, 99, 98, 97, 96 and 95th percentiles and highlight the brighter central structures visible in each filter.

\subsection{Spectroscopy}

\subsubsection{Hen 2-155}

The central star of Hen 2-155 was observed on the night of March 192013 with FORS2 mounted on the ESO-VLT's Antu telescope (Appenzeller et al. 1998). The instrument set-up consisted of a longslit measuring $0.5^{\prime \prime} \times 6.8^{\prime}$, the GRIS_1400V grism and a mosaic of two unbinned $4 \mathrm{k} \times 2 \mathrm{k}$ MIT/LL CCDs, offering a spectral resolution of roughly $0.7 \AA$ with a spectral range of $\sim 4560-5860 \AA$. The observations consisted of 13 contiguous exposures of 900 -s followed by a further exposure of 1200-s. The frames were de-biased and flat-fielded in IRAF ${ }^{3}$. We then used PAMELA (Marsh 1989) to remove the sky contribution

\footnotetext{
3 IRAF is distributed by the National Optical Astronomy Observatories.
}

and obtain the 1D spectra by means of optimal extraction (Horne 1986) as implemented in PAMELA.

The wavelength scale was obtained from one arc spectrum taken during the FORS2 daytime standard calibration routines. We checked for any instrument flexure by monitoring the stability of the nebular [O III] $\lambda 4959$ emission line. This nebular line was slowly shifting with time, so we removed this trend from each individual spectrum accordingly. This wavelength calibration process and the radial velocity analysis presented below were carried out in MOLLY ${ }^{4}$.

The nebula of Hen 2-155 was observed again with the VLT-FORS2 on the night of March 14 2014, with an exposure time of 1200-s employing the GRIS_1200B grism

4 Tom Marsh's MOLLY package is available at http://deneb. astro. warwick. ac.uk/phsaap/software/molly/html/INDEX. html 
(3660 $\AA<\lambda<5110 \AA$ ), directly followed by a 120 -s exposure taken with the GRIS_1200R grism and a GG435 filter $(5750 \AA<$ $\lambda<7310 \AA$ ). Both spectra were taken using a $0.7^{\prime \prime} \times 6.8^{\prime}$ slit (at a position angle of $40^{\circ}$, along the major axis of the nebula) and binning of $2 \times 2$ (providing a resolution of approximately $1.5 \AA$ ). Standard STARLINK routines were employed to bias subtract, flat field correct, wavelength and flux calibrate the spectra (flux calibration was performed using observations of the standard star LTT4816 taken on the same nights and using the same instrumental setups; Hamuy et al. 1992).

\subsubsection{Hen 2-161}

The central star of Hen 2-161 was observed on 31 May 2012 with the queue-scheduled Southern African Large Telescope (SALT; Buckley et al. 2006; O'Donoghue et al. 2006). The Robert Stobie Spectrograph (RSS; Burgh et al. 2003; Kobulnicky et al. 2003) was used with the PG1300 grating and $1.5^{\prime \prime} \times 8^{\prime}$ slit to obtain a 2150-s exposure covering $\lambda=$ 4278-6360 ̊, at a mean resolution of $4.1 \AA$ and a reciprocal dispersion of $0.66 \AA$ pixel $^{-1}$. Basic reductions were applied using the PySALT ${ }^{5}$ package (Crawford et al. 2010). Cosmic ray events were cleaned using the LACOSMIC package (van Dokkum 2001). Wavelength calibration of the contemporaneous Argon arc lamp exposure was performed using standard IRAF tasks IDENTIFY, REIDENTIFY, FITCOORDS and TRANSFORM by identifying the arc lines in each row and applying a geometric transformation to the data frames. The one dimensional spectrum of the central star was extracted using APALL and no nebular subtraction was made.

VLT-FORS2 observations of Hen 2-161 and its central star were acquired on the night of March 14 2014, with an exposure time of 120-s, employing the GRIS_1200R grism and a GG435 filter, and on April 1 2014, with an exposure time of 1140-s and the GRIS_1200B grism (for further details of the setup see Sect. 2.2.1). All spectra were taken using a $0.7^{\prime \prime} \times$ $6.8^{\prime}$ slit (at a position angle of $45^{\circ}$, chosen to cover the major axis of the nebula). Standard STARLINK routines were employed reduce the spectra just as described in Sect. 2.2.1.

\section{Analysis}

\subsection{Hen 2-155}

\subsubsection{Lightcurve and radial velocities}

A Lomb-Scargle analysis was performed in order to determine the periodicity of the variability displayed by the central star of Hen 2-155 using the PERIOD package of the STARLINK software suite (Dhillon et al. 2001). Figure 4 shows the data folded on the ephemeris determined by the analysis,

$\min \mathrm{HJD}=2455985.7865( \pm 0.0001)+0.148275( \pm 0.000008) E$

where min HJD is heliocentric Julian date of the $\mathrm{H} \beta$-continuum lightcurve minimum.

The lightcurve shows smooth sinusoidal variation of peakto-peak amplitude $\sim 0.15 \mathrm{mag}$, with eclipses at phase 0 and 0.5 . The primary eclipse (as the secondary passes in front of the primary), at phase 0 , is approximately 0.2 mag deep, while the secondary eclipse measures approximately $0.05 \mathrm{mag}$ deep. The "v-shaped" eclipse profiles are typical of grazing

\footnotetext{
5 http://pysalt.salt.ac.za
}

Table 4. Excerpt of the log of the radial velocity measurements of the central star of Hen 2-155.

\begin{tabular}{rcc}
\hline \hline Julian date & $\begin{array}{c}\text { Radial velocity } \\
\left(\mathrm{km} \mathrm{s}^{-1}\right)\end{array}$ & $\begin{array}{c}\text { Uncertainty on radial velocity } \\
\left(\mathrm{km} \mathrm{s}^{-1}\right)\end{array}$ \\
\hline 2456371.7604575 & -27.9696 & 2.8980 \\
2456371.7720789 & -50.7702 & 3.4633 \\
2456371.7837002 & -63.7826 & 3.3738 \\
2456371.7953216 & -53.4388 & 3.7784 \\
2456371.8069431 & -29.0446 & 3.2967 \\
\hline
\end{tabular}

Notes. Full table available in machine-readable format at the CDS.

eclipses, indicating a moderate orbital inclination (high inclinations would lead to total eclipses which have a "flat-bottomed" profile). Given that at least one of the stars should be a postAGB remnant (having ejected the PN), then the overall sinusoidal variability is likely to arise from the varying projection of the face of the secondary which is being heated by the hot post-AGB star (known as an "irradiation effect" or "reflection effect"). Given the short period, this variability could also be attributed to one or both stars being close to filling their Roche lobes and, as such, presenting a variable surface area throughout the orbit (known as "ellipsoidal modulation"), this effect is usually associated with rarer, double-degenerate binaries (where both components are post-AGB; Hillwig et al. 2010; Bruch et al. 2001; Santander-García et al. 2015). However, "ellipsoidal modulation" presents two peaks per period with any eclipses occurring at the minima, therefore as the curve shows only one peak per period the origin of the overall variability must originate from the irradiation of the secondary by the primary.

The spectra of Hen 2-155 (Fig. 6) show absorption lines associated with a hot, post-AGB star (e.g. O v $\lambda 5114 \AA$ ) as well as high-excitation emission lines from the nebula (e.g. [Ar IV] $\lambda 4711+4740 \AA$ ). The spectra also show emission lines which have previously been identified as typical of an irradiated mainsequence secondary star (C IV $\lambda 5801+5812 \AA$ ).

Radial velocity measurements, of the primary component of the central star of Hen 2-155, were made via a cross-correlation of the individual O V $\lambda 5114 \AA$ absorption profiles in the VLTFORS2 spectra, and using the average profile as a template. In producing this template, the radial velocities of the O V $\lambda 5114 \AA$ line were first measured by fitting Gaussians to the individual profiles. This preliminary radial velocity curve was then fit with a sine function and the measured orbital motion removed from each individual spectrum using this sine fit. Finally, the resulting profiles were averaged in order to obtain the template for crosscorrelation. This procedure provides a cross-correlation template with good signal-to-noise ratio and a line width representative of that of the individual profiles. The final measured radial velocities (shown folded in the lower panel of Fig. 4 and listed in Table 4) agree extremely well with the ephemeris determined from the photometry, clearly showing that the photometric period is also the orbital period. The variation is well fit by a sinusoid of amplitude $59.6 \pm 1.5 \mathrm{~km} \mathrm{~s}^{-1}$, indicating an extremely low orbital eccentricity (as would be expected for such a short period system). The relatively low amplitude (given the short period) of the variability strongly indicates that either: the orbital inclination is low (as the amplitude varies with the sine of the inclination), or, the mass ratio, $q$, is small (as the amplitude varies with $\left.(1+q)^{-2 / 3}\right)$. As the lightcurve does show shallow eclipses, it is most likely a combination of the two effects that results in the observed amplitude. 

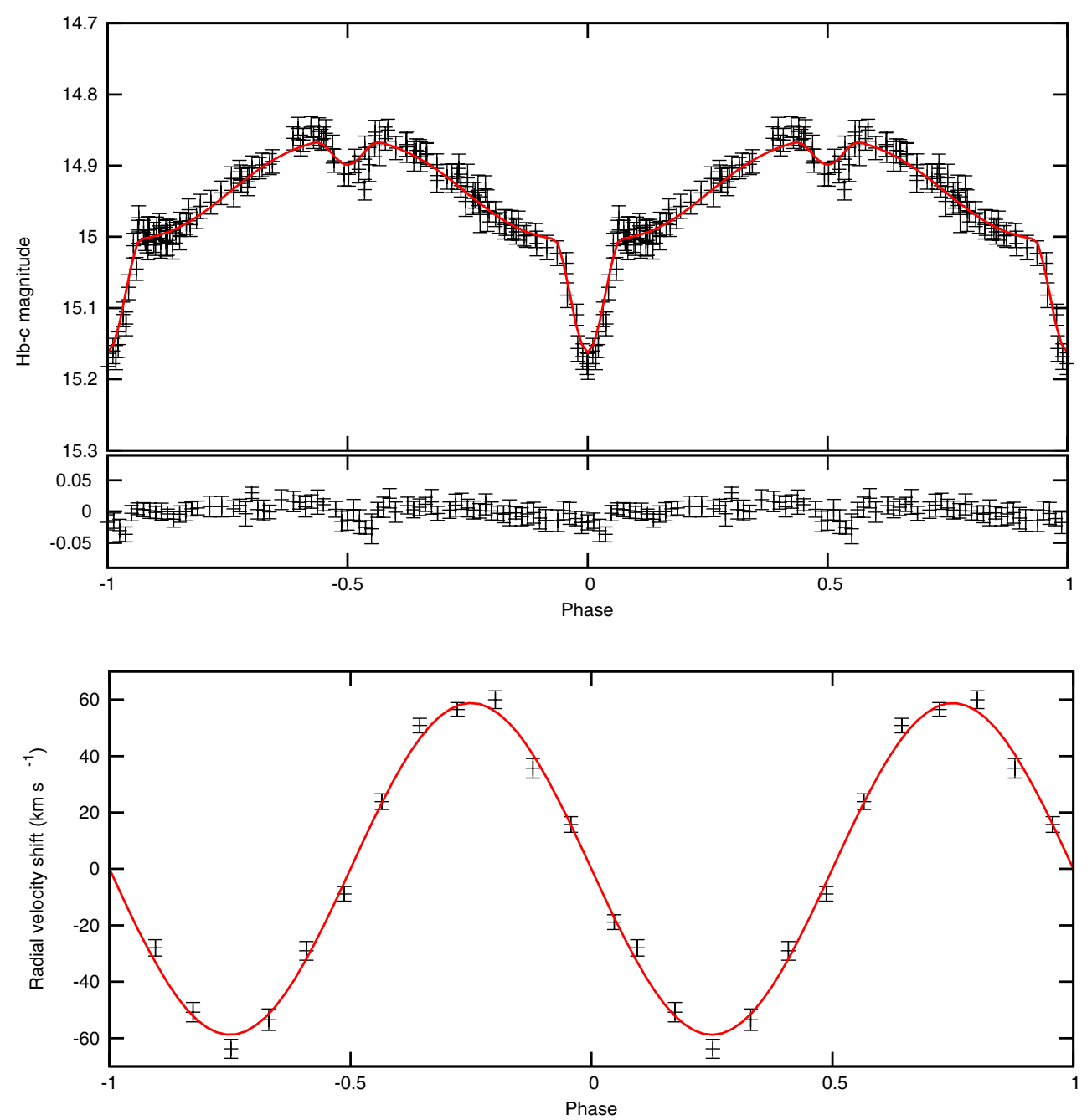

Fig. 4. Folded NTT-EFOSC $2 \mathrm{H} \beta$-continuum photometry of the central star of Hen 2-155 with the NIGHTFALL model overlaid in red (upper panel). Binned residuals between the observed photometry and model are shown in the middle panel. Note that the size of the points represents the photometric uncertainty and contain no estimate of the uncertainty due to variable nebular contamination (which can be significant, at certain phases). The lower panel shows the radial velocity measurements of the central star of Hen 2-155 with the predicted radial velocity curve from the NIGHTFALL model overlaid in red.

All other lines visible in the spectra, including emission lines which may be associated with the secondary of the system (e.g. N III $\lambda 4634+4641 \AA$ and C IV $\lambda 5801+5812 \AA$ ), were also inspected for radial velocity shifts. All but the C IV complex at $\sim 5800 \AA$ were found to be non-variable or too weak to be measured (e.g. the possible detection of O VI $\lambda 5291 \AA$ ). These lines were found to move roughly in phase with the $\mathrm{O} \mathrm{V}$ absorption (a phase shift of 0.037 is calculated from the fit, shown in Fig. 5) but with a lower amplitude $\left(\sim 35 \mathrm{~km} \mathrm{~s}^{-1} \mathrm{cf} . \sim 60 \mathrm{~km} \mathrm{~s}^{-1}\right)$. The radial velocities measured from the $\mathrm{C}$ IV lines, using the same cross-correlation technique as for the $\mathrm{O} v \lambda 5114 \AA$ absorption feature (described above), are shown folded on the orbital period, along with a sinusoidal fit, in Fig. 5 and listed in Table 5. The lower amplitude appears difficult to reconcile with the measured radial velocities of the $\mathrm{O} v$ absorption feature, which must originate from the surface of the primary star. The most likely explanation is that these lines form in the wind of the hot primary and as such trace a region above the WD surface but which has to be restricted to a zone more-or-less between the two stars (as emission from an isotropic wind would present the same radial velocity variability as lines originating from the stellar surface). That the emission is restricted to a relatively small region
Table 5. Excerpt of the log of the radial velocity measurements of the C IV lines of Hen 2-155.

\begin{tabular}{rcc}
\hline \hline Julian date & $\begin{array}{c}\text { Radial velocity } \\
\left(\mathrm{km} \mathrm{s}^{-1}\right)\end{array}$ & $\begin{array}{c}\text { Uncertainty on radial velocity } \\
\left(\mathrm{km} \mathrm{s}^{-1}\right)\end{array}$ \\
\hline 2456371.7604575 & -20.5399 & 2.7514 \\
2456371.7720789 & -22.5216 & 2.6793 \\
2456371.7837002 & -35.5964 & 3.2085 \\
2456371.7953216 & -38.9358 & 2.9990 \\
2456371.8069431 & -24.4214 & 2.5088 \\
\hline
\end{tabular}

Notes. Full table available in machine-readable format at the CDS.

may be indicative of a "hot spot" on the surface of the primary (Deschamps et al. 2013).

An unusual profile is found around the He II $\lambda 4686 \AA$ nebular emission line (the trailed spectra are shown around this line in Fig. 7). As is common in spectra of CSPN, the bright nebular emission line is still visible (even after a basic background subtraction, due to its bright and irregular nature) on top of a broad absorption profile moving in phase with the $\mathrm{O} \mathrm{V}$ absorption line and with a compatible amplitude (we do not measure its 


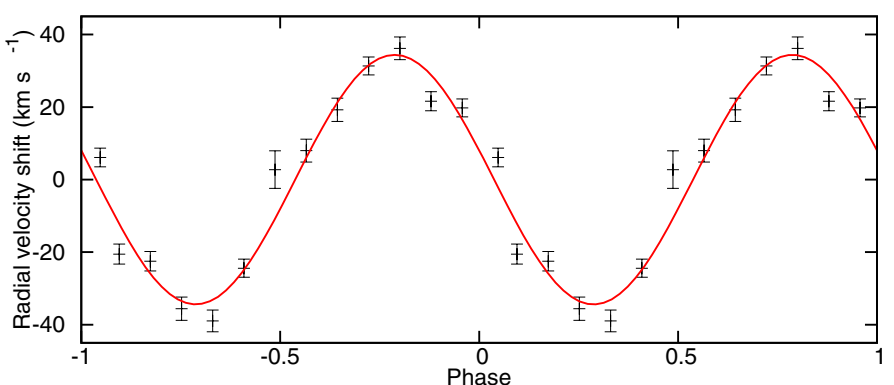

Fig. 5. Radial velocity curve from the C IV lines in Hen 2-155.

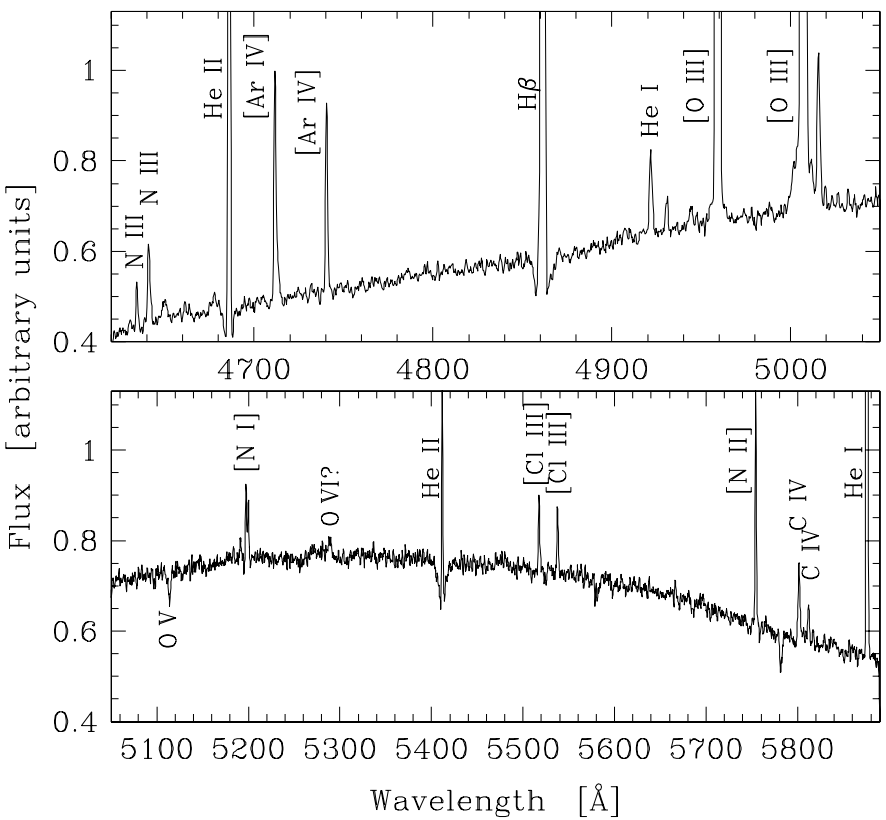

Fig. 6. An example VLT-FORS2 spectrum of Hen 2-155 and its central star.

amplitude due to the obvious complication of the nebular emission), but at phases of approximately 0.25 and 0.75 there is evidence of high velocity emission in anti-phase with the lines originating from the primary. Given the strong contamination from both the nebula and primary, it is not possible to disentangle this emission, and therefore determine its origin and possible association with the secondary.

To properly constrain the parameters of the system, simultaneous modelling, of both the radial velocities (from the $\mathrm{O} v$ line) and lightcurve, was performed using the NIGHTFALL code ${ }^{6}$. All parameters were varied over a wide range of physical solutions, with the final model being selected for having the lowest $\chi^{2}$ fit. Detailed reflection was employed in the modelling (with 5 iterations) in order to properly treat the irradiation of the secondary by the primary. A model atmosphere ${ }^{7}$ was used for the lower temperature (secondary) component with solar metallicity and $\log g$ of 4.5 (Kurucz 1993). The final model lightcurve is shown, along with the residuals to the binned data, in Fig. 4 and the model parameters outlined in Table 6 .

The model represents an excellent fit, with residuals smaller than the uncertainties of individual observations for almost all data points (the scatter of the radial velocity measurements

\footnotetext{
6 http://www.hs.uni-hamburg.de/DE/Ins/Per/Wichmann/ Nightfall.html

7 http://kurucz.harvard.edu/grids/gridPQ0/ip00k2 .pck19
}

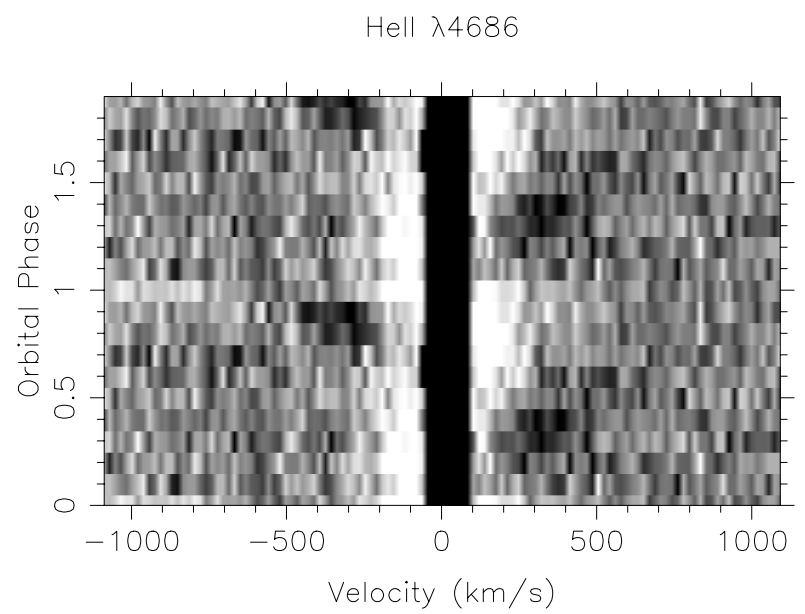

Fig. 7. Trailed spectra showing the emission around the He II $\lambda 4686 \AA$ nebular line. Emission is shown in black, and absorption in white.

Table 6. Modelled and observed binary parameters for Hen 2-155.

\begin{tabular}{rcc}
\hline \hline & Primary & Secondary \\
\hline$T_{\text {eff }}(\mathrm{K})$ & $90000 \pm 5000$ & $3500 \pm 500$ \\
Radius $\left(R_{\odot}\right)$ & $0.31 \pm 0.02$ & $0.30 \pm 0.03$ \\
$\log g$ & - & $4.5^{a}$ \\
\hline Inclination & $68.8^{\circ} \pm 0.8^{\circ}$ \\
$q={ }^{M_{2}} / M_{1}$ & $0.21_{-0.02}^{+0.05}$ \\
$M_{\text {tot }}\left(M_{\odot}\right)$ & $0.75 \pm 0.05$ \\
Period (day) & $0.148275 \pm 0.000008$ \\
\hline
\end{tabular}

Notes. ${ }^{(a)}$ A fixed parameter in the modelling.

around the model curve is greater than the photometric data, but still generally a good fit). The modelled progenitor star lies on the stellar evolutionary tracks of both Bloecker (1995, with an He burning remnant) and Vassiliadis \& Wood (1994, an $\mathrm{H}$ burning remnant with initial mass, $M_{i}=1.0 M_{\odot}$, and metallicity, $Z=0.001$ ) for a remnant mass of $0.625 M_{\odot}$ and $0.623 M_{\odot}$, respectively, both in excellent agreement with the modelled mass of $0.62 M_{\odot}$ (see Fig. 8). The post-AGB age is roughly 10000 years for both of these tracks, which is a reasonable age for a PN and, for a distance of $\sim 3 \mathrm{kpc}$ (Zhang 1995; Phillips 2004; Stanghellini et al. 2008), would imply an expansion velocity of $\sim 30 \mathrm{~km} \mathrm{~s}^{-1}$ (again, perfectly reasonable for a PN, e.g.; Jones et al. 2010a, 2012). It is worth noting that the model parameters also lie close to tracks from both authors with different parameters (mass, $\mathrm{H}$ or He burning, metallicity, etc.), giving an age range from $\sim 3000$ years up to $\sim 25000$ years. Neither end of this expansive range would be out of the question for a PN, however they would imply an exceptionally large or particularly low expansion velocity at the lower and upper age limits, respectively. This range is perhaps reflective of the uncertainty in the starting point for post-AGB tracks, as well as the fact that this is a post-CE system (rather than a single star evolving off the AGB with no external influences, as considered by the models).

The model luminosity (defined by the Boltzmann law, and the model radius and temperature) gives an apparent $V_{0}$ for the central star of $\sim 14.7 \mathrm{mag}$ (assuming a reasonable bolometric correction of -7 ; Reed 1998). From the single $i$-band observation taken at a phase of 0.67 (we choose not to use the $V$-band observation due to the extremely high levels of nebula contamination), 


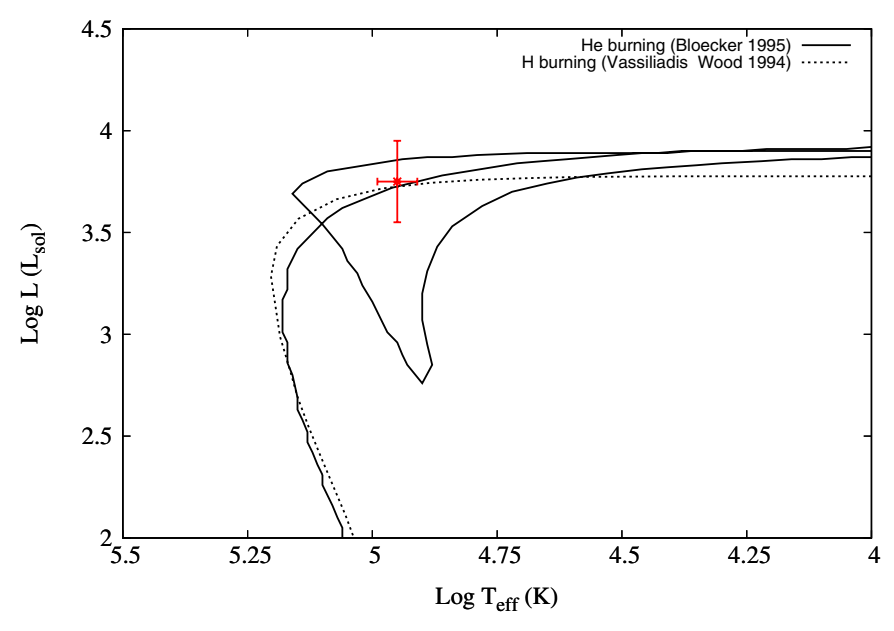

Fig. 8. Temperature and luminosity of the model central star of Hen 2155 overlaid on top of evolutionary tracks for a remnant of mass $0.625 M_{\odot}$ taken from Bloecker (1995) and $0.623 M_{\odot}$ taken from Vassiliadis \& Wood (1994).

we measure an extinction-corrected $V_{0}$ of $\sim 14.4$ mag (uncorrected $V_{0}$ of $\left.15.6 \mathrm{mag}\right)^{8}$. At first this would appear inconsistent, however, noting that the contribution from the secondary is near maximum at this phase, the non-negligible nebular contamination, as well as the uncertainties on the assumed values (particularly the distance), the two values are most certainly consistent and provide a valuable check of the model.

The model temperature of the primary star is, at $90 \mathrm{kK}$, in relatively poor agreement with those in the literature determined by the Zanstra method $(51 \pm 10 \mathrm{kK}$ and $73 \pm 10 \mathrm{kK}$ for He I and He II Zanstra temperatures, respectively; Kaler \& Jacoby 1991). This is to be expected because the Zanstra method depends on accurate measurement of broadband central star magnitudes which will, of course, be contaminated by the contribution from the companion star (as seen in Jones et al. 2014a). Furthermore, the Zanstra temperature determinations assume an optically thick nebula, which would not necessarily be the case for such a hot central star. As such, the Zanstra temperatures can only be considered lower limits for the true central star temperature. The poor agreement between the Zanstra temperatures calculated from hydrogen and helium emission lines, further indicates that these temperatures may not be reflective of the true central star temperature. Kaler \& Jacoby (1991) found a strong correlation between the intensity of the nebular [O III] $\lambda 5007 \AA$ line and the central star temperature, and via their analytical relationship the Stoy Temperature of the central star would be expected to be $\sim 90000 \mathrm{~K}$, in good agreement with the modelled temperature. Furthermore, the presence of $\mathrm{O}$ VI emission, in the VLT-FORS2 spectrum of the central star, indicates a high stellar temperature, probably around $\sim 100000 \mathrm{~K}$ (much higher than this and the $\mathrm{O} V$ lines become much less pronounced, lower and the $\mathrm{O}$ VI line would not be present).

The modelled secondary mass and temperature of $0.13 M_{\odot}$ and $3500 \mathrm{~K}$ are in rough agreement with those predicted by zero age main sequence (ZAMS) models for an M5V star (the ZAMS temperature of a $0.13 M_{\odot}$ star with solar metallicity is approximately $3000 \mathrm{~K}$; Paxton et al. 2011), here the slightly elevated temperature could be partly due to the uncertainty on the model

\footnotetext{
8 Extinction correction performed assuming the extinction law of Howarth (1983), a $c(\mathrm{H} \beta)$ of 0.74 (see Sect. 3.1.2), and $(V-I)_{\mathrm{O}}=-0.40$ (Ciardullo et al. 1999).
}

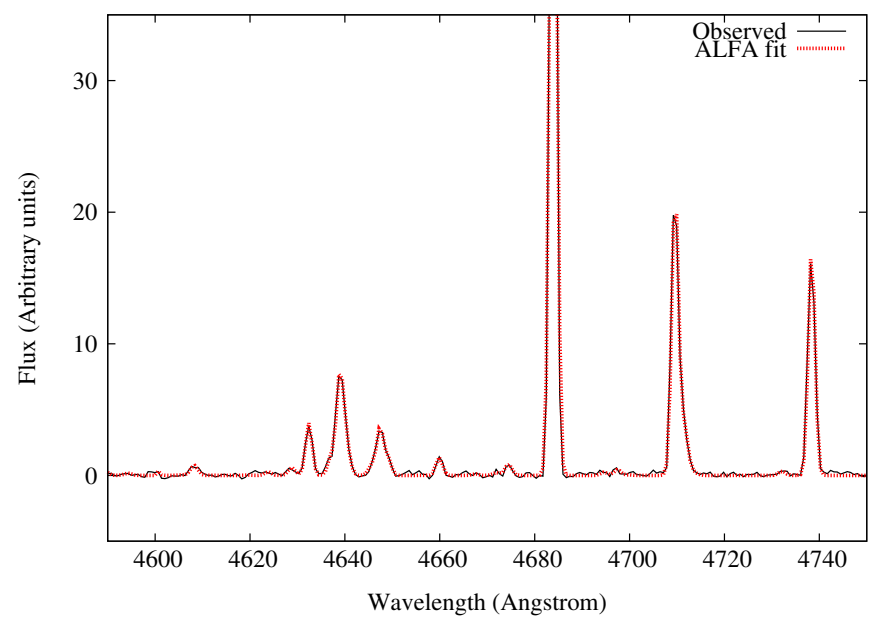

Fig. 9. Continuum-subtracted observations and ALFA fit for a subsection of the FORS2 GRIS_1200B spectrum of the nebula Hen 2-155, covering the $\mathrm{C}$ III and $\mathrm{N}$ III recombination lines as well as several lines of the V1 O II multiplet, which are critical in determining the nebular adf.

temperature as well as the fact that the high levels of irradiation from the primary may cause a global increase in temperature rather than just an increase on the "day side" of the secondary. The modelled radius, however, is much greater than expected for a main sequence star of this mass (almost a factor of two larger, more in keeping with a star of mass $0.3 M_{\odot}$ ). This "inflation" could be associated with the high levels of irradiation from the primary, but could also be a symptom of mass transfer just before entering the CE phase. Further discussion of this "inflation" shall be reserved for Sect. 4 .

The orbital plane, at an inclination of $68.8^{\circ} \pm 0.8^{\circ}$, is expected to be perpendicular to the nebular symmetry axis (Jones et al. 2014b; Nordhaus \& Blackman 2006), while the nebula appears to be viewed more or less side-on (implying an inclination of $\sim 90^{\circ}$ for the binary orbital plane). However, it is not possible to determine the nebular orientation without detailed spatio-kinematic modelling (which necessitates the acquisition of high-resolution, spatially-resolved, nebular spectroscopy) and, moreover, $\mathrm{PNe}$ with similar projections have been found to have inclinations that would be generally consistent with the modelled inclination (for example, HaTr 4 appears similarly side-on but, in fact, has an inclination of $\sim 75^{\circ}$; Tyndall et al. 2012).

\subsubsection{Ionic and total abundances of Hen 2-155}

Apertures containing nebular emission from Hen 2-155 were extracted from the VLT-FORS2 spectroscopy described in Sect. 2. From the resulting one-dimensional spectra, emission line fluxes were derived using the ALFA code (Wesson, in prep.; Jones et al., in prep.), which optimises the parameters of Gaussian fits to the line profiles using a genetic algorithm, fitting all lines simultaneously after subtracting a globally-fitted continuum (see Fig. 9 for a demonstration of the quality of the fit provided by ALFA). These emission line fluxes were then used to derive abundances using the NEAT code (Wesson et al. 2012). This code corrects for interstellar extinction using measured Balmer line ratios, and then determines temperatures and densities from the standard diagnostics. Elemental abundances are then calculated as the flux-weighted averages of the emission lines from each species, 
Table 7. Excerpt from a table of observed, $F(\lambda)$, and dereddened, $I(\lambda)$, nebular emission line fluxes from Hen 2-155 and Hen 2-161.

\begin{tabular}{llcccccccc}
\hline \hline & \multicolumn{4}{c}{ Hen2-155 } & \multicolumn{3}{c}{ Hen2-161 } \\
$\lambda(\AA)$ & Ion & \multicolumn{2}{c}{$F(\lambda)$} & \multicolumn{2}{c}{$I(\lambda)$} & \multicolumn{2}{c}{$F(\lambda)$} & \multicolumn{2}{c}{$I(\lambda)$} \\
\hline 4609.44 & O II & 0.03 & \pm 0.01 & 0.03 & \pm 0.01 & 0.13 & \pm 0.02 & 0.15 & \pm 0.02 \\
4610.20 & O II & 0.06 & \pm 0.01 & 0.06 & \pm 0.01 & \multicolumn{2}{c}{-} & \multicolumn{2}{c}{-} \\
4630.54 & N II & 0.06 & \pm 0.02 & 0.06 & \pm 0.01 & 0.20 & \pm 0.03 & 0.23 & \pm 0.03 \\
4640.64 & N III & 0.77 & \pm 0.02 & 0.85 & \pm 0.02 & 0.53 & \pm 0.02 & 0.61 & \pm 0.02 \\
4647.42 & C III & 0.08 & \pm 0.01 & 0.09 & \pm 0.01 & \multicolumn{2}{c}{-} & \multicolumn{2}{c}{-} \\
\hline
\end{tabular}

Notes. Full table is available in machine-readable format at the CDS.

Table 8. Extinction, temperatures, and densities as determined by the empirical analysis using the NEAT code.

\begin{tabular}{rcc}
\hline \hline & Hen 2-155 & Hen 2-161 \\
\hline$c\left(\mathrm{H}_{\beta}\right)$ & $0.74 \pm 0.01$ & $1.21 \pm 0.01$ \\
$T_{e}([\mathrm{~N} \mathrm{II}])(\mathrm{K})$ & - & $9800 \pm 360$ \\
$T_{e}([\mathrm{O} \mathrm{III}])(\mathrm{K})$ & $11660 \pm 40$ & $8190 \pm 110$ \\
$n_{e}([\mathrm{O} \mathrm{II}])\left(\mathrm{cm}^{-3}\right)$ & $1300 \pm 70$ & $1870 \pm 110$ \\
$n_{e}([\mathrm{~S} \mathrm{II}])\left(\mathrm{cm}^{-3}\right)$ & $1390 \pm 55$ & $1500 \pm 89$ \\
$n_{e}([\mathrm{Ar} \mathrm{IV}])\left(\mathrm{cm}^{-3}\right)$ & $670 \pm 160$ & - \\
\hline
\end{tabular}

and total chemical abundances using the ionisation correction scheme of Delgado-Inglada et al. (2014). At each stage, uncertainties are robustly propagated using a monte carlo technique.

Measured and dereddened fluxes (along with their $1 \sigma$ uncertainties) for Hen 2-155 are shown in Table 7, while the determined nebular properties are listed in table 8 . The reddening is found to be $c\left(\mathrm{H}_{\beta}\right)=0.74 \pm 0.01$ in relatively good agreement with the values found in the literature (0.81, Cavichia et al. 2010; 0.98-1.01, Cahn et al. 1992), as are the individual line fluxes c.f. those presented by Cavichia et al. (2010).

The final ionic and total abundances for Hen 2-155 are shown in Tables 9 and 10, respectively. The abundances indicate that Hen 2-155 is a non-type I PNe (based on the $\mathrm{He} / \mathrm{H}$ and N/O ratios; Peimbert 1978; Kingsburgh \& Barlow 1994). Furthermore, comparison of Oxygen abundances determined from collisionally excited lines (CELs) and optical recombination lines (ORLs), show a strong discrepancy - known as an abundance discrepancy factor $(a d f)$ - whereby the abundance from ORLs is a factor of 6 greater than that from CELs. Such discrepancies are well known, appearing in analyses of all photoionised nebulae, but are generally found to be only a factor of a few (1.5-3 in H II regions, while only $\sim 20 \%$ of PNe are found to have adfs > 5; García-Rojas \& Esteban 2007; Wesson et al. 2005). Further discussion of this enhanced $a d f$ is reserved for Sect. 4

\subsubsection{Photoionisation modelling of Hen $2-155$}

In order to confirm that a hot $(T \sim 90 \mathrm{kK})$ central star, predicted by our modelling of the photometric light curve, is consistent with the overall ionisation state of the nebula, we constructed a simple photoionisation model for comparison to our measured emission line fluxes. It is important to note that our model is only intended to show that the ionisation state of the nebula is consistent with the central star temperature determined from the simultaneous modelling of light- and radial velocity curves. It is not intended to provide a realistic description of the nebula, given that the nebula is clearly both irregular and filamentary (Fig. 3) and that we have no information about surface abundances in
Table 9. Ionic abundances, relative to hydrogen (in the form $\frac{\text { ion }}{\mathrm{H}}$ ), as determined by the empirical analysis using the NEAT code.

\begin{tabular}{|c|c|c|}
\hline Ion & Hen 2-155 & Hen 2-161 \\
\hline $\mathrm{C}^{2+}$ (ORLs) & $3.39 \times 10^{-4^{+1.9}}-1.8 \times 10^{-5} 0^{-5}$ & $2.13 \times 10^{-3}+4.4 \times 10^{-5}-4 \times 10^{-5}$ \\
\hline $\mathrm{C}^{3+}$ (ORLs) & $1.51 \times 10^{-4_{-1.1 .7}^{+1.6} \times 10^{-5}}$ & $2.75 \times 10^{-3}+3.4 \times 10^{-4} 4 \times 10^{-4}$ \\
\hline $\mathrm{N}^{+}$ & $5.27 \times 10^{-6_{-1.1 .8}^{+1.8} \times 10^{-8}}$ & $7.89 \times 10^{-6+8.1 \times 10^{-7}}-7.3 \times 10^{-7}$ \\
\hline $\mathrm{O}^{+}$ & 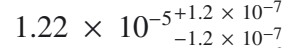 & $1.52 \times 10^{-5}+2.7 \times 10^{-6} \times 10^{-6}$ \\
\hline $\mathrm{O}^{2+}$ (CELs) & $1.43 \times 10^{-4_{-2.2 .9}^{+2.9} \times 10^{-6}}$ & $2.05 \times 10^{-4}+1.2 \times 10^{-5}=10^{-5}$ \\
\hline $\begin{array}{r}\mathrm{O}^{2+}(\mathrm{ORLs}) \\
\operatorname{adf}\left(\mathrm{O}^{2+}\right)\end{array}$ & $\begin{array}{c}9.01 \times 10^{-4+3.8 \times 10^{-5}} \\
6.3 .6 \times 10^{-5}\end{array}$ & $\begin{array}{c}2.26 \times 10^{-3}+8.5 \times 10^{-5}-8.2 \times 10^{-5} \\
11.0^{-5}\end{array}$ \\
\hline $\mathrm{Ne}^{2+}$ & $5.07 \times 10^{-5}+6.7 \times 10^{-7}$ & $7.75 \times 10^{-5}+4.2 \times 10^{-6}$ \\
\hline $\mathrm{Ar}^{2+}$ & $8.76 \times 10^{-7}{ }_{-4.5}^{+4.7} \times 10^{-8}$ & $1.66 \times 10^{-6}{ }_{-6.2 \times 10^{-8}}$ \\
\hline $\mathrm{Ar}^{3+}$ & $3.91 \times 10^{-7}+5.6 \times 10^{-9} 9 \times 10^{-9}$ & $1.87 \times 10^{-7}+2.4 \times 10^{-8}-2 \times 10^{-8}$ \\
\hline $\mathrm{S}^{+}$ & $2.44 \times 10^{-7+2.0 \times 10^{-9}}+2.0 \times 10^{-9}$ & $2.76 \times 10^{-7+2.6 \times 10^{-8}}-2.4 \times 10^{-8}$ \\
\hline $\mathrm{S}^{2+}$ & $1.83 \times 10^{-6}{ }_{-5.5 \times 10^{+5.7}}^{-8} \times 10^{-8}$ & $2.50 \times 10^{-6+3.4 \times 10^{-7}}-3.0 \times 10^{-7}$ \\
\hline
\end{tabular}

Notes. All abundances are calculated using CELs unless otherwise indicated.

Table 10. Total nebular abundances from Hen 2-155 and Hen 2-161, determined using the NEAT code.

\begin{tabular}{|c|c|c|}
\hline Element & Hen 2-155 & Hen 2-161 \\
\hline $\mathrm{H}$ & 1.00 & 1.00 \\
\hline $\mathrm{He}$ & $0.11_{-6.5 \times 10^{-4}}^{+6.5 \times 10^{-4}}$ & $0.13_{-8.3 \times 10^{-4}}^{+7.7 \times 10^{-4}}$ \\
\hline $\mathrm{C}$ (ORLs) & $4.91 \times 10^{-4}+2.5 \times 10^{-5}$ & $4.87 \times 10^{-3}{ }_{-3.3}^{+3.5} \times 10^{-4}$ \\
\hline $\mathrm{N}$ & $7.01 \times 10^{-5} 5_{-1.4}^{+1.5 \times 10^{-6}} \times 10^{-6}$ & $1.14 \times 10^{-4}+8.9 \times 10^{-6}$ \\
\hline $\mathrm{O}$ & $1.62 \times 10^{-4+3.0 \times 10^{-6}}+2.9 \times 10^{-6}$ & $2.21 \times 10^{-4+1.2 \times 10^{-5}}-1.1 \times 10^{-5}$ \\
\hline $\mathrm{Ne}$ & $5.74 \times 10^{-5+6.7 \times 10^{-7}}-6.6 \times 10^{-7}$ & $8.35 \times 10^{-5}+5.4 \times 10^{-6} .1 \times 10^{-6}$ \\
\hline S & $3.50 \times 10^{-6^{+1.1}}-1 \times 10^{-7} 0^{-7}$ & $4.81 \times 10^{-6^{+6.7}}-5.9 \times 10^{-7}$ \\
\hline $\mathrm{Ar}$ & $1.26 \times 10^{-6}{ }_{-4.6}^{+4.8 \times 10^{-8}}$ & $1.84 \times 10^{-6}{ }_{-7.7}^{+7.8 \times 10^{-8}} \times 10^{-8}$ \\
\hline
\end{tabular}

the central star with which to realistically estimate the ionising spectrum.

We modelled the nebula, using MOCCASIN (Ercolano et al. 2003), as a sphere of radius $10^{18} \mathrm{~cm}$, estimated from the angular extent of the nebula and a distance of $3 \mathrm{kpc}$. The model has uniform hydrogen density of $1000 \mathrm{~cm}^{-3}$ (see previous section), and abundances based on the empirical analysis of observed line fluxes (for a list of the final abundances used see Table 10). The model reproduces fairly well the overall emission spectrum of the nebula (see Fig. 10) despite being so simplistic. The central star in the model is a blackbody with a temperature of $90 \mathrm{kK}$ and a luminosity of $5200 L_{\odot}$, both matching well with 


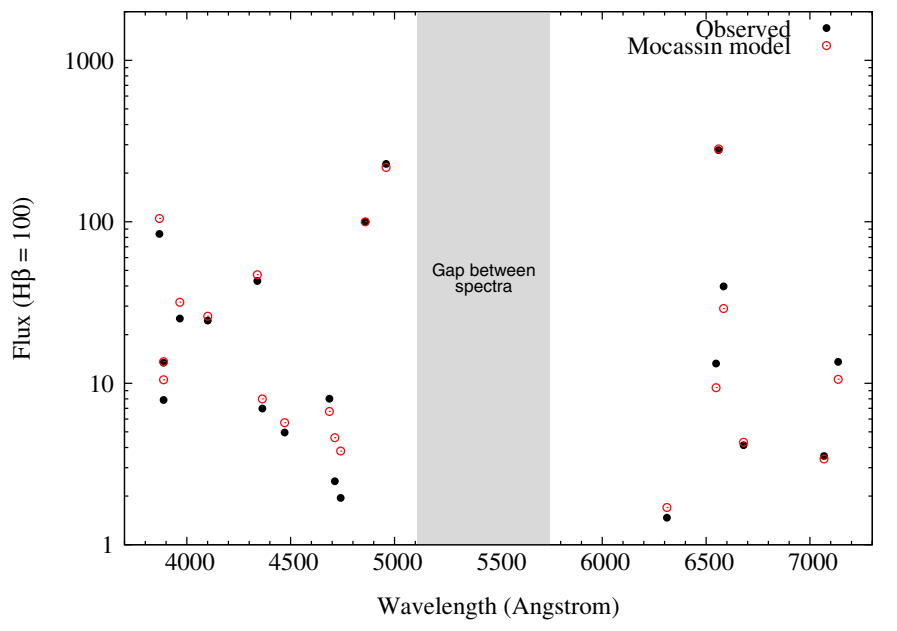

Fig. 10. A comparison of emission line fluxes from the MOCASSIN model of Hen 2-155 and those measured from the FORS2 spectroscopy (Table 7).

the temperature and luminosity determined from simultaneous modelling of the light- and radial velocity curves. A more realistic spectral shape for the ionising source (i.e. not a perfect blackbody) and more realistic density distribution would certainly affect the ionisation balance, but the model clearly shows that a hot central star is consistent with the measured emission line ratios. As such, we conclude that the temperature determined in this work, $90 \mathrm{kK}$, constitutes a more accurate estimate of central star temperature than those made using other methodologies in the literature (e.g. Zanstra temperatures).

\subsection{Hen 2-161}

\subsubsection{Stellar photometry and spectroscopy}

The photometric observations of Hen 2-161 were taken in two different filters - the majority in Gunn- $i$ taken using NTTEFOSC2 with the rest taken in Cousins- $I$ with the SAAO CCD mounted on the SAAO 1.0-m. In order to ensure no colour effects were present in the combination of data taken through two different $I$-band filters, the relative magnitude of several field stars (used in photometering the central star) were measured, with the results generally agreeing within the uncertainties of the individual measurements. As such, we do not apply any colour correction to the photometry of the central star - this may, indeed, induce spurious variability, so the results will be assessed in the context of the combined data and the NTT data alone (as the vast majority of the data originate from this telescope).

A Lomb-Scargle analysis was performed for the complete photometry of the central star of Hen 2-161 using the PERIOD package of the STARLINK software suite (Dhillon et al. 2001). The data show clear variability, but unfortunately any true period remains difficult to ascertain most likely due to it being extremely close to the periodic aliases of nightly observing (12 $\mathrm{h}$ and $1 \mathrm{day}$ ). The Lomb-Scargle periodogram of the data is presented in Fig. 11, highlighting the strength of each of the peaks around each alias. The data show a maximum variation of roughly $0.2 \mathrm{mag}$ ( $0.15 \mathrm{mag}$ excluding the SAAO $1.0-\mathrm{m}$ data), although, given the possible periods, this is not certain to be the true peak-to-peak amplitude of the variability. It is highly unlikely that this variability is spurious, given that it does not correlate with any aspect of the observing parameters other than the time of the observation (spurious variability due to nebular

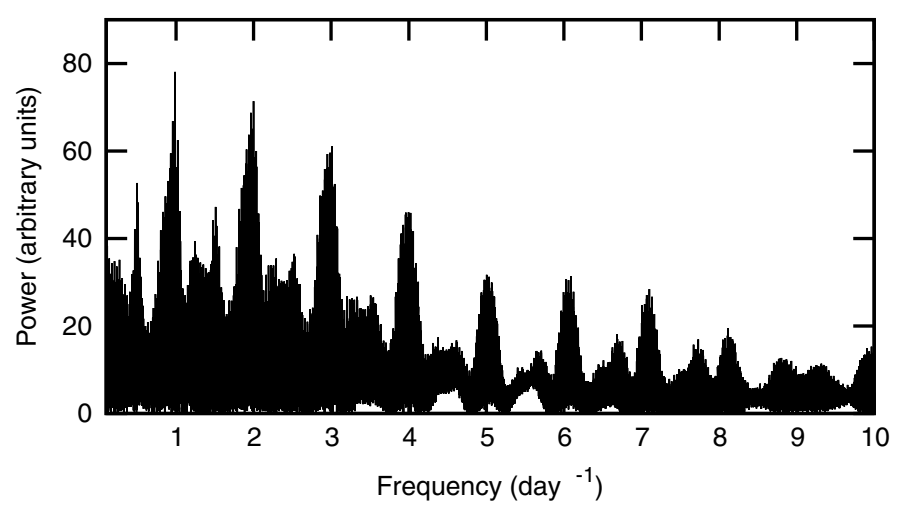

Fig. 11. Lomb-Scargle periodogram of the photometric observations of Hen 2-161.

contamination or colour effects generally can be found to correlate with the seeing or airmass at the time of the observation; Jones 2011). Even if the inclusion of the SAAO 1.0-m data is erroneous, there is still a clear upward trend in the data obtained with EFOSC2-NTT (see Fig. 12).

The VLT-FORS2 spectra and SALT-RSS spectrum all show absorption features associated with a hot post-AGB nebular progenitor (e.g. He II $\lambda 4542 \AA$, He II $\lambda 5412 \AA, \mathrm{N}$ V $\lambda 4604 \AA$ ) as well as emission lines frequently, but not exclusively (see above discussion of these lines in the spectra of Hen 2-155), associated with irradiated, main-sequence companions (see Fig. 13, and Miszalski et al. 2011b; Corradi et al. 2011). Both absorption and emission features visible in all spectra were inspected for velocity shifts, with respect to nebular emission lines (e.g. $\mathrm{H} \beta$, He I $\lambda 4472 \AA$, [O III] $\lambda 4959 \AA$ ), with all radial velocities agreeing within the uncertainties of the measurements (which, given the low resolution of the spectrum, are rather large for this purpose). This is, perhaps, not unreasonable given that, for a period of $\sim 1$ day, the spectra would have all taken at around the same phase which may have been close to conjunction where any radial velocity shifts would be minimal (this is consistent with the phases of the highly speculative sine curve overlaid in Fig. 12 where all spectra would have been taken at phase $\sim 0$ ). However, this does mean that we cannot rule out that the variability of the central star is not associated with binarity, but, given the persistent nature of the observed photometric variability (pulsations often vary on timescales shorter than the 2 years over which our observations span, particularly in such a young post-AGB object; Winget \& Kepler 2008), the presence of emission lines associated with an irradiated secondary, and the offcentre central star (often associated with central star binary; Soker \& Rappaport 2001; Jones et al. 2010b), we conclude that Hen 2-161 is most likely host to a close binary central star with a possible orbital period of approximately 1 day. We strongly encourage follow-up observations, particularly at other longitudes, in order to confirm and further constrain its nature as a post-CE binary.

\subsubsection{Ionic and total abundances of Hen 2-161}

Just as for Hen 2-155 (Sect. 3.1.2), nebular apertures of Hen 2-161 were extracted from the VLT-FORS2 spectroscopy described in Sect. 2 and processed using the ALFA and NEAT codes. The measured and dereddened fluxes (along with their $1 \sigma$ uncertainties) for Hen 2-161 are shown in Table 7, while the determined properties of the nebula are listed in Table 8 . The 

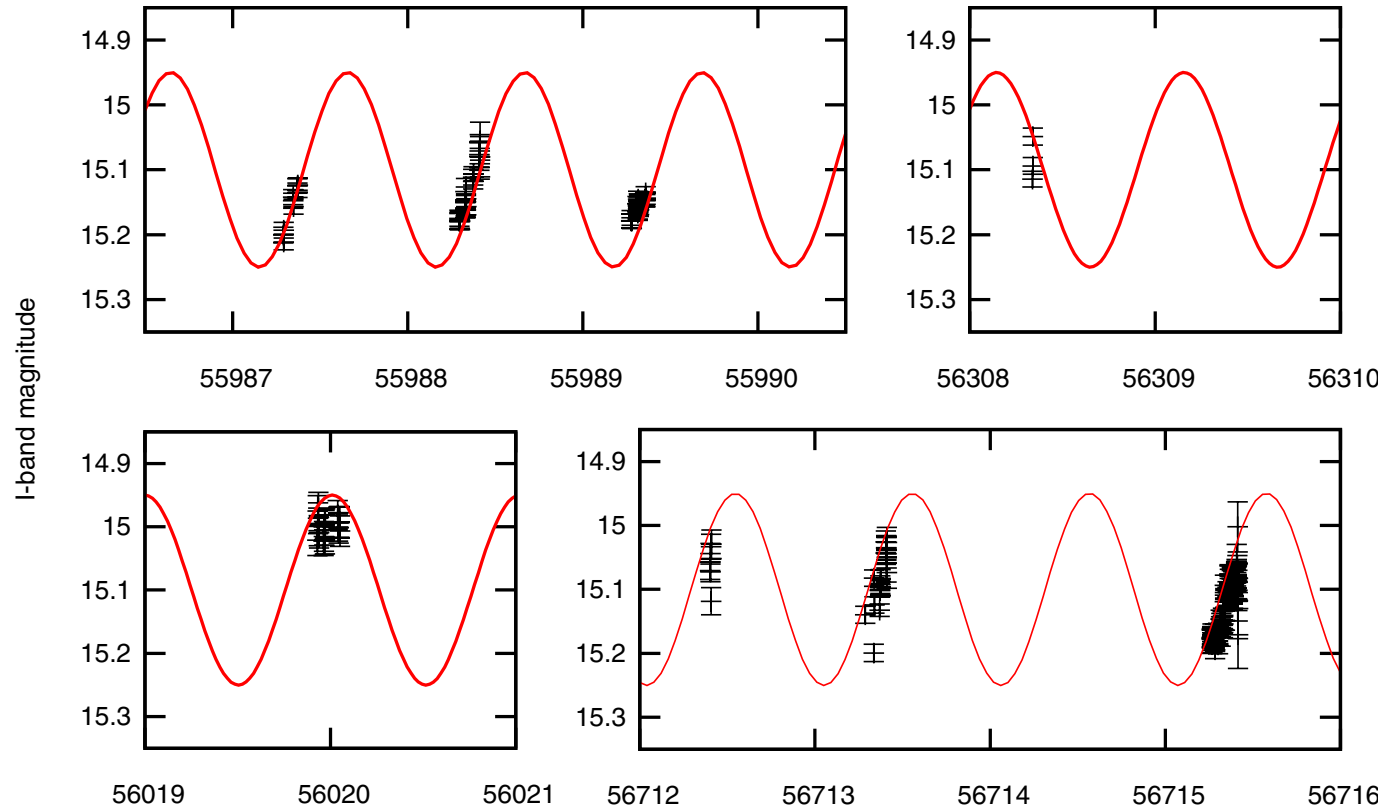

MJD

Fig. 12. $I$-band photometry of the central star of Hen 2-161 with an overlaid sinusoid of period 1.011 day (the peak of the periodogram shown in Fig. 11). The observations from the SAAO 1.0-m telescope (taken in a different $I$-band filter to the rest of the observations) are shown in the lower left panel, highlighting that even without adding the data from this telescope, the variability is still clear. The scatter in the points may be due to an error in the period or to variable nebula contamination as seen in Miszalski et al. (2011b).

reddening is found to be $c\left(\mathrm{H}_{\beta}\right)=1.21 \pm 0.01$, while the literature values range from $0.35-1.80$ (Cahn et al. 1992), depending on the method used in its determination (the upper value is determined from the ratio of radio $5 \mathrm{GHz}$ flux density and $\mathrm{H} \beta$, while the lower is from the ratio of $\mathrm{H} \alpha$ and $\mathrm{H} \beta$ ). Given that our measurement is the weighted average of all the Balmer line ratios available in the covered wavelength range (which covers $\mathrm{H} \alpha$ through to $\mathrm{H} \zeta$ ), and the results are consistent across all ratios (as reflected by the relatively small uncertainty), it is clear that the value measured here must represent the most accurate determination of the reddening to date.

The final ionic and total abundances are shown in Tables 9 and 10 , respectively. Hen 2-161 shows systematically higher heavy element abundances than Hen 2-155, just about satisfying the general criterion for Type I PNe $(\mathrm{He} / \mathrm{H} \geq 0.125, \mathrm{~N} / \mathrm{O} \geq 0.5$ Peimbert 1978) $)^{9}$. This may be indicative of a higher mass progenitor or that the binary evolution has had little (or lessened) effect on the chemical evolution of the primary (De Marco 2009). However, just as for Hen 2-155, Hen 2-161 displays a large $a d f$ (over 10), possibly typical of central star binarity (see Sect. 4).

\section{Discussion}

The central star of Hen 2-155 is a photometrically variable binary star with a period of 0.148 day, while the central star of Hen 2-161 has also been shown to be photometrically variable with a possible period of $\sim 1$ day. While a $\sim 1$ day period is highly suspicious, one other bCSPN is known to have a period extremely close to $\sim 1$ day (Abell 65, Shimansky et al. 2009; Bond \& Livio 1990) meaning that such a period should not be ruled out. However, it is ultimately unclear whether the photometric variability in the central star of Hen 2-161 is associated with binarity, but we conclude that it is the most likely cause and

\footnotetext{
9 Note that Kingsburgh \& Barlow (1994) proposed a higher threshold for being Type I which Hen 2-161 does not satisfy (N/O $\geq 0.8$ ).
}

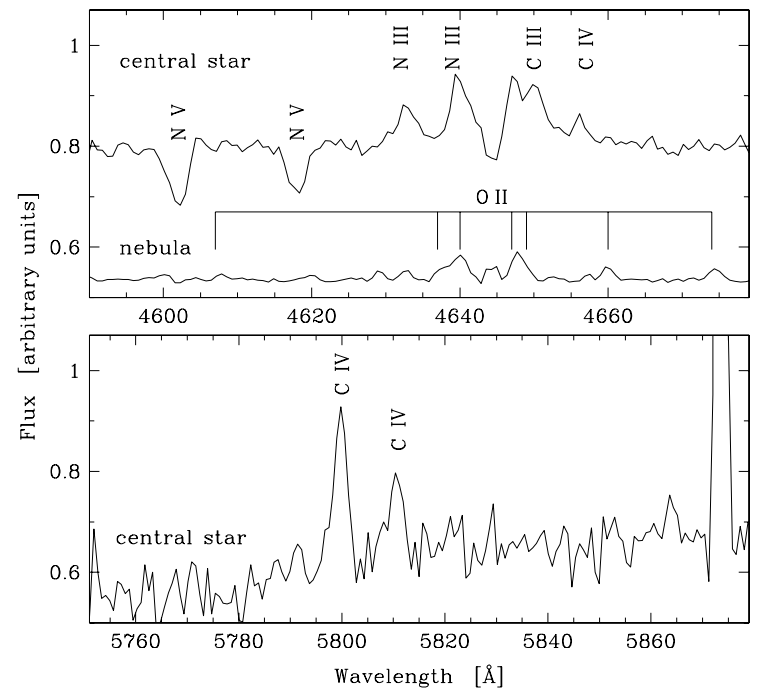

Fig. 13. VLT-FORS2 spectroscopy of the central star of Hen 2-161 displaying both absorption features from a hot post-AGB central star and emission line complexes frequently associated with an irradiated, mainsequence secondary. In both panels, the central star spectrum is shown without any nebula subtraction. In the upper panel, an extraction of the nebula close to the central star is displayed (with an arbitrary offset) in order to highlight that the emission lines originate from the central star and not from the nebula.

encourage follow-up observations (both photometric and spectroscopic) in order to fully reconstruct any orbit. Given its brightness $(I \sim 15.1 \mathrm{mag})$ and southern declination, it should be easily accessible with available telescopes, the main constraint comes in the form of seeing as, due to a bright star approximately $2^{\prime \prime}$ away from the CSPN (to the south, more or less on the edge of the nebular ring, see Fig. 2), good seeing is needed to ensure photometry of the central star alone. 
Table 11. Binary CSPNe from the literature where parameters for both primary and secondary stars have been derived from simultaneous modelling of photometric and radial velocity observations.

\begin{tabular}{|c|c|c|c|c|c|c|c|c|}
\hline Nebula & $\begin{array}{c}\text { Period } \\
\text { (day) }\end{array}$ & $\begin{array}{c}M_{\mathrm{CS}} \\
\left(M_{\odot}\right) \\
\end{array}$ & $\begin{array}{l}R_{\mathrm{CS}} \\
\left(R_{\odot}\right) \\
\end{array}$ & $\begin{array}{c}T_{\mathrm{CS}} \\
(\mathrm{kK}) \\
\end{array}$ & $\begin{array}{c}M_{\mathrm{S}} \\
\left(M_{\odot}\right)\end{array}$ & $\begin{array}{c}R_{\mathrm{S}} \\
\left(R_{\odot}\right) \\
\end{array}$ & $\begin{array}{c}T_{\mathrm{S}} \\
(\mathrm{kK})\end{array}$ & Notes \\
\hline Abell $46^{a}$ & 0.47 & $0.51 \pm 0.05$ & $0.15 \pm 0.02$ & $49.5 \pm 4.5$ & $0.15 \pm 0.02$ & $0.46 \pm 0.02$ & $3.9 \pm 0.4$ & Double-lined \\
\hline Abell $63^{a}$ & 0.47 & $0.63 \pm 0.05$ & $0.35 \pm 0.01$ & $78 \pm 3$ & $0.29 \pm 0.03$ & $0.56 \pm 0.02$ & $6.1 \pm 0.2$ & Double-lined \\
\hline Abell $65^{b}$ & 1.00 & $0.56 \pm 0.04$ & $0.056 \pm 0.008$ & $110 \pm 10$ & $0.22 \pm 0.04$ & $0.41 \pm 0.05$ & $5.0 \pm 1.0$ & Not eclipsing \\
\hline $\mathrm{BE} \mathrm{Uma}^{c}$ & 2.29 & $0.70 \pm 0.07$ & $0.08 \pm 0.01$ & $105 \pm 5$ & $0.36 \pm 0.07$ & $0.72 \pm 0.05$ & $5.8 \pm 0.3$ & Double-lined \\
\hline Ds $1^{d}$ & 0.36 & $0.63 \pm 0.03$ & $0.16 \pm 0.01$ & $77 \pm 3$ & $0.23 \pm 0.01$ & $0.40 \pm 0.01$ & $3.4 \pm 1$ & Not eclipsing, double-lined \\
\hline Hen $2-428^{e}$ & 0.18 & $0.88 \pm 0.13$ & $0.68 \pm 0.04$ & $32.4 \pm 5.2$ & $0.88 \pm 0.13$ & $0.68 \pm 0.04$ & $30.9 \pm 5.2$ & Double-lined \\
\hline NGC $6026^{f}$ & 0.53 & $0.57 \pm 0.05$ & $1.06 \pm 0.05$ & $38 \pm 3$ & $0.57 \pm 0.05$ & $0.05 \pm 0.01$ & $146 \pm 15$ & - \\
\hline
\end{tabular}

Notes. The upper five have main-sequence companions, while the lower two are shown to be double-degenerates.

References. (a) Afşar \& Ibanoğlu (2008); (b) Hillwig et al. (2015); (c) Ferguson et al. (1999); (d) Hilditch et al. (1996); (e) Santander-García et al. (2015); (f) Hillwig et al. (2010).

The central star of Hen 2-155 was also found to be a singlelined, radial-velocity variable with a period and ephemeris consistent with that derived from the photometric observations. Simultaneous modelling of the lightcurve and radial velocity curve shows that the system consists of a hot nebular progenitor, with a temperature of $\sim 90 \mathrm{kK}$, and a much cooler companion. The modelled primary mass, radius and temperature are consistent with evolutionary tracks and the ionisation state of the nebula. The modelled secondary has a much larger radius, and slightly higher temperature, than expected for its mass. It is worthy of note, that there is an indication of emission from the secondary star in the system, but that its radial velocity in this line could not be measured due to low signal-to-noise and contamination from spectral features of the primary and nebula.

Radial velocity measurements of the C IV $\lambda 5801+5812 \AA$ emission lines present in the spectra of Hen 2-155 show variability in phase with that of the $\mathrm{O} v$ absorption lines originating from the primary, but with a lower amplitude. The true origin of this emission is quite uncertain, but it is most likely to originate from the wind of the hot primary and hence trace a region above its surface. Its restriction to a region between the two stars may be associated with a "hot spot" on the surface of the primary from which the wind is enhanced (Deschamps et al. 2013). A "hot spot" would be in keeping with the Roche-lobe filling nature of the secondary, whereby direct impact of mass transferred from the secondary onto the surface of the primary would be responsible for its formation (no accretion disk would be formed because the primary is larger than the circularisation radius for any material transferred onto it). Given the faintness of these lines, and that no other lines display the same behaviour, we conclude that while of great interest and warranting further investigation, this unusual line profile gives no reason to doubt the stellar parameters derived from the modelling.

Only a handful of other binary CSPNe have had their parameters derived by in-depth studies similar to the one presented here ${ }^{10}$ (with the results summarised in Table 11). Of those seven

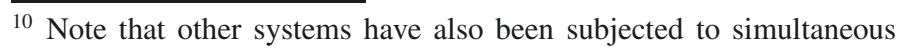
modelling of spectroscopic and photometric observations but, due to their non-eclipsing nature (and the lack of multiband photometry), their parameters (especially radii) could not be derived (e.g. HFG 1; Exter et al. 2005). Similarly, other systems have been modelled based on photometry alone, but here masses can only be estimated via indirect means (e.g. Hen 2-11; Jones et al. 2014a). It is also worth noting that the majority of the other systems listed in Table 11 are double-lined spectroscopic binaries, meaning that a more direct constraint can be placed on their mass ratios than in the case of a single-lined binary (as is the case for Hen 2-155, where the parameter is constrained by the modelling).
}

systems, two are found to be double-degenerates where both stars have evolved to the post-AGB phase, while the other five are found to have secondaries which are greatly inflated with respect to the ZAMS radii for their given masses.

The origin of the inflation seen in the secondaries of CSPNe is unclear, but several possibilities have been considered in the literature. Afşar \& Ibanoğlu (2008) considered that the inflated secondaries in Abell 46 and Abell 63 may originate from magnetic activity, ruling this possibility out due to the also increased temperatures (magnetic activity has been shown to result in increased radii and reduced temperatures). De Marco et al. (2008) state that the extreme heating of the secondaries by the much hotter primaries could lead to them "puffing up" as the star is irradiated by leakage of radiation from the "day side" to the "night side", the level of this effect is, however, unclear and may not contribute sufficiently to increase the radii by the levels seen. This hypothesis is, however, supported by the elevated temperatures determined both from the modelling and from spectroscopic observations of the "night side" of the secondaries (De Marco et al. 2008). Finally, the inflation could be associated with mass from the primary being accreted just prior to or during the CE phase, knocking the secondary out of thermal equilibrium. For fully convective secondaries (or those with deep convective envelopes), the amount of matter required to be accreted in order to produce the observed inflation is minimal, but the accretion rate does need to be significant $\left(\gtrsim 10^{-5} M_{\odot} \mathrm{yr}^{-1}\right.$; Prialnik \& Livio 1985). Hydrodynamic models indicate that the spiral-in speeds during the $\mathrm{CE}$ are highly supersonic, leading to the formation of a bow shock and preventing any material from being accreted by the secondary during the CE phase (Sandquist et al. 1998). However, recently it has been suggested that there could be a period of pre-CE mass transfer which, due to the extremely short nature of the $\mathrm{CE}$, could be responsible for the secondaries being out of thermal equilibrium even after exiting the CE.

The central star of the Necklace is a post-CE binary where the secondary shows clear evidence of being chemically polluted by AGB material from the primary (Miszalski et al. 2013; Boffin 2014a), proving that mass transfer can and does occur in these systems (and possibly in significant quantities $-0.03-0.35 M_{\odot}$ in the case of the Necklace). This mass transfer almost certainly occurred immediately prior to the $\mathrm{CE}$, also being responsible for forming the polar jets seen in the nebula (the dynamical ages of the jets are older than that of the central regions of the nebula, which is expected to be the remnant of the ejected CE, Corradi et al. 2011). Interestingly, amongst the other nebulae shown to play host to inflated secondaries, there is a second case where the polar regions of the nebula appear to have been ejected prior 
to the CE (Abell 63, Mitchell et al. 2007). Estimates of the mass transfer rate during the formation of the jets in these objects are of order $10^{-5}-10^{-4} M_{\odot} \mathrm{yr}^{-1}$ (Tocknell et al. 2014), almost certainly sufficient to produce the inflation found in the secondaries. It is worth noting that, while the other nebulae are found to host inflated secondaries, including Hen 2-155, they have yet to be shown to present evidence of polar outflows formed prior to the CE phase. This may just be due to a lack of detailed morphokinematical study ${ }^{11}$ or simply because these outflows are generally extremely light and may since have simply dissipated into the surroundings and are no longer visible.

In this study, both Hen 2-155 and Hen 2-161 are found to display sub-solar, non-type I abundances (Hen 2-161 could be considered a marginal Type I) and higher than average $a d f s$, both of which have possible links to binarity. Non-type I abundances can arise naturally from low-mass single star progenitors or high mass progenitors where a binary interaction has cut short the chemical evolution of the progenitor (De Marco 2009) - given that both nebulae display type I-like morphologies, this may be an indication that the latter scenario has played a role (however, this cannot be confirmed with the current observations). Perhaps a stronger link to binarity is the high $a d f$, where recent work has shown that the PNe with the highest known adfs all host a central binary star (Corradi et al. 2015, Jones et al. in prep; and references therein). High $a d f s$ also strengthen the link between PNe with binary central stars and other binary phenomena, such as novae, which are also known to display extremely high $a d f s$ (Wesson et al. 2003, 2008).

We strongly encourage the detailed study of other post-CE CSPN and their host nebulae (as well as the continued search for new systems) in order to improve the statistics and to establish the parameter space for future modelling and study of the $\mathrm{CE}$ and its effect on PN formation and evolution. In the immediate future, this work should focus on radial velocity studies and modelling of eclipsing systems for which high-quality photometric monitoring has already been performed (e.g. M3 16, H 2-29 and M 2-19; Miszalski et al. 2008), as eclipsing systems offer the best prospect for well-constrained parameters. For noneclipsing systems, the greatest uncertainty often arises from the a wide-range of inclinations offering reasonable fits to the data - in this cases, the now well established link between nebular inclination and binary inclination may be used to successfully break the degeneracy. This, however, requires detailed spatiokinematical modelling of the host nebulae (e.g. Tyndall et al. 2012; Huckvale et al. 2013) in order to determine the inclination of the nebular symmetry axis which can then be assumed to be roughly perpendicular to binary orbital plane (Jones et al. 2012, 2014b; Nordhaus \& Blackman 2006).

Acknowledgements. We would like to thank Klaus Werner for his assistance in the interpretation of the stellar spectroscopy, and Tom Marsh for the use of his MOLLY and PAMELA software packages. D.J. would like to thank Jorge García-Rojas for useful discussions regarding the interpretation of the nebular spectroscopy. This paper is based on observations made with ESO Telescopes at the La Silla Paranal Observatory under programme IDs 088.D-0573, 090.D0435, 091.D-0475, 092.D-0449 \& 093.D-0038. This paper includes observations made with the Southern African Large Telescope (SALT) under programme 2012-1-RSA-009, and with the $1 \mathrm{~m}$ telescope of the South African Astronomical Observatory (SAAO). Based on observations made with the NASA/ESA Hubble Space Telescope, and obtained from the Hubble Legacy Archive, which is a collaboration between the Space Telescope Science Institute (STScI/NASA),

\footnotetext{
11 This may likely be the case for Hen 2-155 given the presence of pairs geometrically opposed knots. Further kinematical study will be essential not only in constraining the morphology of Hen 2-155 but also the nature of these "jet" candidates.
}

the Space Telescope European Coordinating Facility (ST-ECF/ESA) and the Canadian Astronomy Data Centre (CADC/NRC/CSA). This research made use of NASA's Astrophysics Data System; the SIMBAD database, operated at CDS, Strasbourg, France; the VizieR catalogue access tool, CDS, Strasbourg, France. This research has been supported by the Spanish Ministry of Economy and Competitiveness (MINECO) under grants AYA2012-38700 and AYA201235330. P.R.G. acknowledges support from the MINECO under the Ramón y Cajal programme (RYC-2010-05762). Finally, we wish to thank the anonymous referee for their comments which have improved the detail of this manuscript.

\section{References}

Afşar, M., \& Ibanoğlu, C. 2008, MNRAS, 391, 802

Appenzeller, I., Fricke, K., Fürtig, W., et al. 1998, The Messenger, 94, 1

Bertin, E., \& Arnouts, S. 1996, A\&AS, 117, 393

Bloecker, T. 1995, A\&A, 299, 755

Boffin, H. M. J. 2014a, in Ecology of Blue Straggler Stars (Springer)

Boffin, H. M. J. 2014b, in ASP proceedings of the 19th European White Dwarf Workshop

Bond, H. E., \& Livio, M. 1990, ApJ, 335, 568

Boffin, H. M. J., Miszalski, B., \& Jones, D. 2012a, A\&A, 545, A146

Boffin, H. M. J., Miszalski, B., Rauch, T., et al. 2012b, Science, 338, 773

Bond, H. E. 2000, in Asymmetrical Planetary Nebulae II: From Origins to Microstructures, eds. J. H. Kastner, N. Soker, \& S. Rappaport, ASP Conf. Ser., 199, 115

Bruch, A., Vaz, L. P. R., \& Diaz, M. P. 2001, A\&A, 377, 898

Buckley, D. A. H., Swart, G. P., \& Meiring, J. G. 2006, in SPIE Conf. Ser., 6267,0

Burgh, E. B., Nordsieck, K. H., Kobulnicky, H. A., et al. 2003, in SPIE Conf. Ser. 4841, eds. M. Iye, \& A. F. M. Moorwood, 1463

Buzzoni, B., Delabre, B., Dekker, H., et al. 1984, The Messenger, 38, 9

Cahn, J. H., Kaler, J. B., \& Stanghellini, L. 1992, A\&AS, 94, 399

Cavichia, O., Costa, R. D. D., \& Maciel, W. J. 2010, Rev. Mex. Astron. Astrophys., 46, 159

Ciardullo, R., Bond, H. E., Sipior, M. S., et al. 1999, AJ, 118, 488

Corradi, R. L. M., Sabin, L., Miszalski, B., et al. 2011, MNRAS, 410, 1349

Corradi, R. L. M., García-Rojas, J., Jones, D., \& Rodríguez-Gil, P. 2015, ApJ, 803,99

Crawford, S. M., Still, M., Schellart, P., et al. 2010, in SPIE Conf. Ser., 7737

De Marco, O. 2009, PASP, 121, 316

De Marco, O., Hillwig, T. C., \& Smith, A. J. 2008, AJ, 136, 323

Delgado-Inglada, G., Morisset, C., \& Stasińska, G. 2014, MNRAS, 440, 536

Deschamps, R., Siess, L., Davis, P. J., \& Jorissen, A. 2013, A\&A, 557, A40

Dhillon, V. S., Privett, G. J., \& Duffey, K. P. 2001, Starlink User Note 167.6 (Rutherford Appleton Laboratory)

Durand, S., Acker, A., \& Zijlstra, A. 1998, A\&AS, 132, 13

Epchtein, N., Deul, E., Derriere, S., et al. 1999, A\&A, 349, 236

Ercolano, B., Barlow, M. J., Storey, P. J., \& Liu, X.-W. 2003, MNRAS, 340, 1136

Exter, K. M., Pollacco, D. L., Maxted, P. F. L., Napiwotzki, R., \& Bell, S. A. 2005, MNRAS, 359, 315

Ferguson, D. H., Liebert, J., Haas, S., Napiwotzki, R., \& James, T. A. 1999, ApJ, 518,866

García-Rojas, J., \& Esteban, C. 2007, ApJ, 670, 457

Hamuy, M., Walker, A. R., Suntzeff, N. B., et al. 1992, PASP, 104, 533

Henize, K. G. 1967, ApJS, 14, 125

Hilditch, R. W., Harries, T. J., \& Hill, G. 1996, MNRAS, 279, 1380

Hillwig, T. C., Bond, H. E., Afşar, M., \& De Marco, O. 2010, AJ, 140, 319

Hillwig, T. C., Frew, D. J., Louie, M., et al. 2015, ApJ, 150, 30

Horne, K. 1986, PASP, 98, 609

Howarth, I. D. 1983, MNRAS, 203, 301

Huckvale, L., Prouse, B., Jones, D., et al. 2013, MNRAS, 434, 1505

Jones, D. 2011, Ph.D. Thesis, Jodrell Bank Centre for Astrophysics University of Manchester

Jones, D., Lloyd, M., Mitchell, D. L., et al. 2010a, MNRAS, 401, 405

Jones, D., Lloyd, M., Santander-García, M., et al. 2010b, MNRAS, 408, 2312

Jones, D., Mitchell, D. L., Lloyd, M., et al. 2012, MNRAS, 420, 2261

Jones, D., Boffin, H. M. J., Miszalski, B., et al. 2014a, A\&A, 562, A89

Jones, D., Santander-García, M., Boffin, H. M. J., Miszalski, B., \& Corradi,

R. L. M. 2014b, in Asymmetrical Planetary Nebulae VI Conference, 43

Kaler, J. B., \& Jacoby, G. H. 1991, ApJ, 372, 215

Kingsburgh, R. L., \& Barlow, M. J. 1994, MNRAS, 271, 257

Kobulnicky, H. A., Nordsieck, K. H., Burgh, E. B., et al. 2003, in SPIE Conf Ser. 4841, eds. M. Iye, \& A. F. M. Moorwood, 1634 
Kurucz, R. L. 1993, VizieR Online Data Catalog: 6/039

Manick, R., Miszalski, B., \& McBride, V. 2015, MNRAS, 448, 1789

Marsh, T. R. 1989, PASP, 101, 1032

Miszalski, B., Acker, A., Moffat, A. F. J., Parker, Q. A., \& Udalski, A. 2008, A\&A, 488, L79

Miszalski, B., Acker, A., Moffat, A. F. J., Parker, Q. A., \& Udalski, A. 2009a, A\&A, 496, 813

Miszalski, B., Acker, A., Parker, Q. A., \& Moffat, A. F. J. 2009b, A\&A, 505, 249

Miszalski, B., Corradi, R. L. M., Boffin, H. M. J., et al. 2011a, MNRAS, 413, 1264

Miszalski, B., Jones, D., Rodríguez-Gil, P., et al. 2011b, A\&A, 531, A158

Miszalski, B., Boffin, H. M. J., \& Corradi, R. L. M. 2013, MNRAS, 428, L39

Mitchell, D. L., Pollacco, D., O'Brien, T. J., et al. 2007, MNRAS, 374, 1404

Nordhaus, J., \& Blackman, E. G. 2006, MNRAS, 370, 2004

O'Donoghue, D., Buckley, D. A. H., Balona, L. A., et al. 2006, MNRAS, 372, 151

Paxton, B., Bildsten, L., Dotter, A., et al. 2011, ApJS, 192, 3

Peimbert, M. 1978, in Planetary Nebulae, ed. Y. Terzian, IAU Symp., 76, 215

Phillips, J. P. 2004, MNRAS, 353, 589

Preite-Martinez, A., Acker, A., Koeppen, J., \& Stenholm, B. 1989, A\&AS, 81, 309

Prialnik, D., \& Livio, M. 1985, MNRAS, 216, 37

Reed, B. C. 1998, JRASC, 92, 36

Sahai, R., Morris, M. R., \& Villar, G. G. 2011, AJ, 141, 134
Sandquist, E. L., Taam, R. E., Chen, X., Bodenheimer, P., \& Burkert, A. 1998, ApJ, 500, 909

Santander-García, M., Rodríguez-Gil, P., Corradi, R. L. M., et al. 2015, Nature, 519,63

Shimansky, V. V., Pozdnyakova, S. A., Borisov, N. V., et al. 2009, Astrophys. Bull., 64, 349

Snodgrass, C., Saviane, I., Monaco, L., \& Sinclaire, P. 2008, The Messenger, 132,18

Soker, N., \& Rappaport, S. 2001, ApJ, 557, 256

Soker, N., Rappaport, S., \& Harpaz, A. 1998, ApJ, 496, 842

Stanghellini, L., Shaw, R. A., \& Villaver, E. 2008, ApJ, 689, 194

Tocknell, J., De Marco, O., \& Wardle, M. 2014, MNRAS, 439, 2014

Tylenda, R., Siódmiak, N., Górny, S. K., Corradi, R. L. M., \& Schwarz, H. E. 2003, A\&A, 405, 627

Tyndall, A. A., Jones, D., Lloyd, M., O’Brien, T. J., \& Pollacco, D. 2012, MNRAS, 422, 1804

van Dokkum, P. G. 2001, PASP, 113, 1420

Vassiliadis, E., \& Wood, P. R. 1994, ApJS, 92, 125

Wesson, R., Liu, X.-W., \& Barlow, M. J. 2003, MNRAS, 340, 253

Wesson, R., Liu, X.-W., \& Barlow, M. J. 2005, MNRAS, 362, 424

Wesson, R., Barlow, M. J., Liu, X.-W., et al. 2008, MNRAS, 383, 1639

Wesson, R., Stock, D. J., \& Scicluna, P. 2012, MNRAS, 422, 3516

Winget, D. E., \& Kepler, S. O. 2008, ARA\&A, 46, 157

Zhang, C. Y. 1995, ApJS, 98, 659 